Annals of Mathematics, 153 (2001), 471-531

\title{
Critical metrics for the determinant of the Laplacian in odd dimensions
}

\author{
By K. OKIKIOLU*
}

\begin{abstract}
Let $M$ be a closed compact $n$-dimensional manifold with $n$ odd. We calculate the first and second variations of the zeta-regularized determinants $\operatorname{det}^{\prime} \Delta$ and $\operatorname{det} L$ as the metric on $M$ varies, where $\Delta$ denotes the Laplacian on functions and $L$ denotes the conformal Laplacian. We see that the behavior of these functionals depends on the dimension. Indeed, every critical metric for $(-1)^{(n-1) / 2} \operatorname{det}^{\prime} \Delta$ or $(-1)^{(n-1) / 2}|\operatorname{det} L|$ has finite index. Consequently there are no local maxima if $n=4 m+1$ and no local minima if $n=4 m+3$. We show that the standard 3-sphere is a local maximum for $\operatorname{det}^{\prime} \Delta$ while the standard $(4 m+3)$-sphere with $m=1,2, \ldots$, is a saddle point. By contrast, for all odd $n$, the standard $n$-sphere is a local extremal for $\operatorname{det} L$.

An important tool in our work is the canonical trace on odd class operators in odd dimensions. This trace is related to the determinant by the formula $\operatorname{det} Q=\mathrm{TR} \log Q$, and we prove some basic results on how to calculate this trace.
\end{abstract}

\section{Contents}

1. Introduction

1.1. The determinant of the Laplacian

1.2. The canonical trace

1.3. Critical metrics

2. Regularization theorems

2.1. Symbols

2.2. Canonical splitting of operators

2.3. Canonical trace for products

*The author was supported by the National Science Foundation and the Alfred P. Sloan Foundation. 
3. Variation formulas

3.1. First variation of $\operatorname{det}^{\prime} \Delta$

3.2. First variation of $\operatorname{det} L$

3.3. Second variation of $\operatorname{det}^{\prime} \Delta$

3.4. Second variation of $\operatorname{det} L$

4. Critical points have finite index

5. Standard spheres which are extremal

5.1. Fourier series for singular kernels

5.2. The hessian of $F$ at $S^{3}$

5.3. The hessian of $\log \operatorname{det} L$ at $S^{n}$

6. Saddle points for $\operatorname{det}^{\prime} \Delta$

\section{Introduction}

In this paper we prove several results about critical metrics for the determinant of the Laplacian. One of our main analytic tools is the canonical trace on odd class operators, and we also present here some basic results on how to compute this trace. In Section 1.1 we give a brief introduction to the determinant of the Laplacian, in Section 1.2 we state results on the canonical trace and in Section 1.3 we state the results on critical metrics for the determinant of the Laplacian.

1.1. The determinant of the Laplacian. Let $M$ be a smooth compact $n$ dimensional manifold without boundary, let $g$ be a Riemannian metric on $M$ and let $\Delta: C^{\infty}(M) \rightarrow C^{\infty}(M)$ be the (positive) Laplacian for the metric $g$. The conformal Laplacian is

$$
L=\Delta+\alpha \mathrm{S}, \quad \alpha=\frac{n-2}{4(n-1)},
$$

where $\mathrm{S}$ denotes the scalar curvature. We will define the zeta regularized determinants $\operatorname{det} L$ and $\operatorname{det}^{\prime} \Delta$.

Let $B: C^{\infty}(M) \rightarrow C^{\infty}(M)$ be a second order elliptic differential operator which is bounded below, with eigenvalues

$$
\lambda_{1} \leq \lambda_{2} \leq \ldots
$$

counted by multiplicity. Define

$$
Z(s)=Z(B, s)=\sum_{\lambda_{j} \neq 0} \lambda_{j}^{-s}
$$

where the branch of $\lambda^{s}$ is chosen so that $\lambda^{s}>0$ when $\lambda>0$ and $s \in \mathbb{R}$. The sum in (1.1.1) converges for $\Re s>n / 2$ by Weyl's law, and can be analytically 
continued to a meromorphic function on $\mathbb{C}$ which is regular at $s=0$; see $[\mathrm{S}]$. When $B$ is invertible, define

$$
\operatorname{det} B=e^{-Z^{\prime}(0)}
$$

which is formally equal to the product of the eigenvalues of $L$. When $B$ is not invertible, define $\operatorname{det} B=0$ and

$$
\operatorname{det}^{\prime} B=e^{-Z^{\prime}(0)} .
$$

For some general theory of zeta-regularized determinants, see [BKF1], [BKF2], [Fo1], [Fo2], [Fo3], [KV], [Ok1], [Ok2].

The zeta regularized determinant of the Laplacian was introduced in [RS] in connection with topology, and the idea was then taken up by physicists in order to formally evaluate Gaussian functional integrals. In particular A. Polyakov applied $\operatorname{det}^{\prime} \Delta$ on compact surfaces to string theory. A few years later, B. Osgood, R. Phillips and P. Sarnak studied the determinant of the Laplacian on surfaces and investigated its applications to spectral geometry, see [OPS1], [OPS2], [OPS3].

THEOREM [OPS1]. If $M$ is a closed surface then of all metrics in a given conformal class and of a given area, the uniform metric has the maximum determinant.

Remarks. 1. All metrics on the 2-sphere are conformally equivalent and so among all metrics on the 2-sphere of area $4 \pi$, the standard 2-sphere maximizes the determinant of the Laplacian. This special case of the result was first proved by Onofri [On].

2. In [OPS2], the functional $\log \operatorname{det}^{\prime} \Delta$ is used to prove that the space of isospectral metrics on a surface is compact in the smooth topology.

In higher dimensions, the functional det $L$ has been studied by T. Branson, A. Chang, M. Gursky, B. Ørsted, J. Qing, P. Yang and others; see [BO1], [BO2], [BCY], [CY], [CQ1], [CQ2], [CQ3], [Gur].

TheOREm [CY]. For the following cases of 4-manifolds $M$, the standard metric is a global maximum for $\operatorname{det} L$ among metrics in the same conformal class and with the same volume:

$S^{4}, \mathbb{C} P^{2}, S^{2} \times S^{2}, \mathbb{R}^{4} / \Gamma$ for any lattice $\Gamma, \mathbb{H}^{2} \times \mathbb{H}^{2} / \Gamma$ for any lattice $\Gamma$, $\mathbb{C} H^{2} / \Gamma$ for any lattice $\Gamma, \Sigma \times S^{2}$ with $\Sigma$ hyperbolic and those Kähler-Einstein surfaces which are not locally symmetric.

Here, we collect some extremal properties of spheres for the functionals $\operatorname{det}^{\prime} \Delta$ and $\operatorname{det} L$. The results can be found in [On], [Ri], [BCY], [Br]. 


\begin{tabular}{|c|c|c|c|c|}
\hline standard & extremal type & for & among metrics fixing & proved by \\
\hline$S^{2}$ & global max & $\operatorname{det}^{\prime} \Delta$ & area & Onofri \\
$S^{3}$ & local max & $\operatorname{det}^{\prime} \Delta$ & volume + conformal class & Richardson \\
$S^{4}$ & global min & $\operatorname{det} L$ & volume + conformal class & Branson-Chang-Yang \\
$S^{6}$ & global max & $\operatorname{det} L$ & volume + conformal class & Branson \\
\hline
\end{tabular}

Remarks. 1. Notice that whether $S^{n}$ is a maximum or a minimum for the conformal Laplacian under conformal deformation depends on the dimension.

2. In odd dimensions, $\operatorname{det} L$ is constant on each conformal class.

3. A. Chang and J. Qing studied $\operatorname{det} L$ for manifolds with boundary. In particular in [CQ2] they consider the conformal Laplacian on the unit ball $B^{4}$ in $\mathbb{R}^{4}$ with Robin boundary conditions on $S^{3}$, and show that for metrics in the conformal class of the standard metric on $B^{4}$ with fixed volume, the standard metric is extremal for $\operatorname{det}^{\prime} L$.

An important tool in proving the 2-dimensional results and the results involving $\operatorname{det} L$ is an explicit formula for the variation of $\log \operatorname{det} L$ under conformal variations of the metric $g_{0}$ which is computable from the local data $g_{0}$ and $\dot{g}(0)$; see [OPS1], [BO1], [Ok2]. On surfaces this is the Polyakov-RaySinger variation formula. Suppose that $g_{0}$ is a fixed metric on the surface $M$. Let $g=e^{2 \phi} g_{0}$ be a conformal metric. Here, $\phi$ is a smooth function on $M$. Write $\Delta_{0}, \Delta$ for the Laplacians in the metrics $g_{0}, g$ respectively, so that $\Delta=e^{-2 \phi} \Delta_{0}$. Then

$$
\begin{array}{r}
\log \operatorname{det}^{\prime} \Delta-\log \operatorname{det}^{\prime} \Delta_{0}=-\frac{1}{6 \pi}\left(\frac{1}{2} \int_{M}\left|\nabla_{0} \phi\right|^{2} d A_{0}+\right. \\
\left.+\int_{M} K_{0} \phi d A_{0}\right) \\
+\log A-\log A_{0}
\end{array}
$$

where $\nabla_{0}$ is the gradient, $d A_{0}$ is the area element, $K_{0}$ is the Gaussian curvature in the metric $g_{0}$ and $A$, respectively $A_{0}$, is the area of $M$ in the metric $g, g_{0}$. These quantities can be computed from $g_{0}$ and $\phi$. In dimension $n>2$, there is no such formula for $\operatorname{det}^{\prime} \Delta$ under conformal variations, or for $\operatorname{det} L \operatorname{or}_{\operatorname{det}^{\prime}} \Delta$ under general variations, which is why these situations have been studied less. Next we state two results that deal with these situations.

THEOREM [Ri]. Let $g_{0}$ be a metric on a compact closed 3-manifold. If $g_{0}$ is a critical point of $\operatorname{det}^{\prime} \Delta$ for metrics in the conformal class of $g_{0}$ with the same volume, and if $-Z(1) \lambda_{1} \geq 5$, then $g_{0}$ is a local maximum for $\operatorname{det}^{\prime} \Delta$ among metrics in this class. The standard metric on the cubic torus is an example of such a metric $g_{0}$. 
Remark. The standard metric $g_{0}$ on the 3 -sphere does not satisfy the condition $-Z(1) \lambda_{1} \geq 5$, but Richardson dealt with this case separately.

TheOREM [Chi]. The determinant of the Laplacian on the space of flat 3-tori of volume 1 has a local maximum at the torus corresponding to the facecentered cubic lattice.

For a Riemannian surface, the functional,

$$
F=\log \operatorname{det}^{\prime} \Delta-\left(1-\frac{\chi(M)}{6}\right) \log A,
$$

is homogeneous of degree zero, where $\chi(M)$ is the Euler characteristic of $M$. Osgood, Phillips and Sarnak noticed that if the metric is constrained to vary within a conformal class then the gradient flow for $F$ is equal to the Ricci flow.

$$
\frac{d g}{d t}=\operatorname{grad} F=-(K-\bar{K}) g
$$

where $K$ is the Gauss curvature and $\bar{K}$ is the average curvature

$$
\bar{K}=\frac{1}{A} \int_{M} K d A .
$$

This flow was studied by R. Hamilton and B. Chow.

Theorem [Ha], [Cho]. Given any initial metric on the closed compact surface $M$, the Ricci flow exists for all time and converges to a metric of constant curvature.

1.2. The canonical trace. We summarize the theory of the canonical trace. Let $M$ be a closed compact $n$-dimensional manifold with $n$ odd, and let $E$ be a complex $N$-dimensional vector bundle over $M$. Write $\mathbb{C}(N)$ for the complex $N \times N$ matrices. Let $Q: C^{\infty}(E) \rightarrow C^{\infty}(E)$ be a polyhomogeneous pseudodifferential operator. Choice of local coordinates identifies a local trivialization of $E$ with $\Omega \times \mathbb{C}^{N}$ where $\Omega \subset \mathbb{R}^{n}$, and the symbol of $Q$ has an expansion of the form

$$
q(x, \xi) \sim q_{d}(x, \xi)+q_{d-1}(x, \xi)+\ldots, \quad|\xi| \rightarrow \infty
$$

where $q, q_{j}$ are $\mathbb{C}(N)$-valued functions on $\Omega$ with $q_{j}(x, t \xi)=t^{j} q_{j}(x, \xi)$ for $t>0$. The Schwartz kernel $K(Q, x, y)$ of $Q$ is smooth away from the diagonal. If $d<-n$ then $Q$ is trace class, $K(Q, x, y)$ is continuous across the diagonal, and the trace of $Q$ is given by the formula

$$
\operatorname{trace} Q=\int_{M} \operatorname{trace} K(Q, x, x)
$$


When $d>-n, K(Q, x, y)$ blows up at the diagonal and $K(Q, x, x)$ and trace $Q$ are undefined. Substitutes for these quantities can often be defined by embed$\operatorname{ding} Q$ in an analytic family of operators $Q(z)$ and then analytically continuing $K(Q(z), x, x)$ and trace $Q(z)$.

Remark. Write $\wedge$ for the bundle of smooth densities on $M$. Away from the diagonal, $K(Q, x, y)$ is a smooth section of $\pi_{1}^{*} E \otimes \pi_{2}^{*}\left(E^{*} \otimes \wedge\right)$ where $\pi_{i}: M \times M \rightarrow M$ is the projection onto the $i^{\text {th }}$ factor. When $d<-n$, $K(Q, x, x)$ is a smooth section of $E \otimes E^{*} \otimes \wedge$, which is a bundle over $M$. If we fix local coordinates $\left(x^{1}, \ldots, x^{n}\right)$ on $M$ then we can identify the density $a(x) d x^{1} \ldots d x^{n}$ with the function $a(x)$, thus trivializing $\wedge$. If we also fix a local trivialization of $E$, then $K(Q, x, x)$ is identified with a $\mathbb{C}(N)$-valued function of the coordinates.

Let $B: C^{\infty}(M) \rightarrow C^{\infty}(M)$ be a positive self-adjoint elliptic differential operator of order $\beta>0$. For $x \in M$ and $\Re z \ll 0$,

$$
K\left(Q B^{z / \beta}, x, x\right)
$$

is well-defined. It extends to a meromorphic function with simple poles. The Guillemin-Wodzicki residue density of $Q$ is the section of $E \otimes E^{*} \otimes \wedge$ given by

$$
\operatorname{RESD}(Q, x):=\left.\operatorname{res}\right|_{z=0} ^{\operatorname{mer}} K\left(Q B^{z / \beta}, x, x\right)
$$

where the notation res $\left.\right|_{z_{0}} ^{\text {mer }} f(z)$ means the residue of the analytic continuation of $f(z)$ at $z=z_{0}$. It is independent of the choice of $B$ and can be computed in local coordinates from the symbol:

$$
\operatorname{RESD}(Q, x)=\frac{1}{(2 \pi)^{n}}\left(\int_{\{|\xi|=1\}} q_{-n}(x, \xi) d \sigma_{n-1}(\xi)\right) d \xi .
$$

Here, the sphere $|\xi|=1$ is defined with respect to any metric on $M$, and $d \sigma_{n-1}$ is surface measure on the sphere; see [Gui], [Ka], [Wo]. Define

$$
\operatorname{RES} Q:=\left.\operatorname{res}\right|_{z=0} ^{\operatorname{mer}} \operatorname{trace} Q B^{z / \beta}=\int_{M} \operatorname{trace} \operatorname{RESD}(Q, x) .
$$

In fact the family $Q B^{z / \beta}$ can be replaced by any analytic family $Q(z)$ where $Q(z)$ has degree $d+z$ and $Q(0)=Q$. From (1.2.2) it is clear that for the following classes of operators, the local residue vanishes identically.

(a) Trace class pseudodifferential operators (that is, operators of order less than $-n$ )

(b) Operators of nonintegral degree

(c) Odd class operators (defined below). 
Definition 1.2.1. Let $q \in C^{\infty}\left(\mathbb{R}^{n} \backslash 0, \mathbb{C}(N)\right)$ be homogeneous of degree $j$. Then $q$ has regular parity if

$$
q(-\xi)=(-1)^{j} q(\xi)
$$

and has singular parity if

$$
q(-\xi)=(-1)^{j+1} q(\xi)
$$

Now suppose that $q$ has an expansion

$$
q(\xi) \sim q_{d}(\xi)+q_{d-1}(\xi)+\ldots, \quad \xi \rightarrow \infty, \quad q_{j}(t \xi)=t^{j} q_{j}(\xi), \quad t>0 .
$$

Then $q$ has regular, respectively singular, parity in $\xi$ as $\xi \rightarrow \infty$ if all of the homogeneous functions $q_{j}$ have regular, respectively singular, parity. Notice that polynomials have regular parity.

Suppose that the dimension $n$ is odd. The odd class operators $Q$ are those classical pseudodifferential operators for which the symbol $q(x, \xi)$ has regular parity in $\xi$ as $\xi \rightarrow \infty$. Such operators $Q$ are also said to satisfy the transmission property. The odd class operators on $E$ form an algebra containing the differential operators and inverses of invertible elliptic operators.

In the cases (a), (b) and (c), it can be shown that

$$
\operatorname{KERD}(Q, x):=\left.K\left(Q B^{z / \beta}, x, x\right)\right|_{z=0} ^{\text {mer }}
$$

is independent of $B$, and hence the canonical trace

$$
\operatorname{TR} Q:=\left.\operatorname{trace} Q B^{z / \beta}\right|_{z=0} ^{\text {mer }}=\int_{M} \operatorname{trace} \operatorname{KERD}(Q, x)
$$

is independent of $B$. In fact the analytic family $Q B^{z / \beta}$ in (1.2.6) can be replaced by any analytic family $Q(z)$ where $Q(z)$ has degree $d+z, Q(0)=Q$, and the symbol $q(z)$ of $Q(z)$ has the expansion

$$
q(z)(x, \xi) \sim q_{d}(z)(x, \xi)+q_{d-1}(z)(x, \xi)+\ldots,
$$

where $q_{j}(z)(x, \xi)$ is homogeneous in $\xi$ of degree $j+z$ and

$$
q_{j}(z)(x,-\xi)=(-1)^{j} q_{j}(z)(x, \xi) .
$$

The canonical trace was introduced by M. Kontseivich and S. Vishik who verified some basic properties; see $[\mathrm{KV}]$.

(A) If $Q$ is trace class then $\operatorname{KERD}(Q, x)=K(Q, x, x)$ and so $\operatorname{TR} Q=\operatorname{trace} Q$.

(B) If $n$ is odd and $\partial$ is a differential operator then $\operatorname{KERD}(\partial, x)=0$ and so $\operatorname{TR} \partial=0$.

(C) If $Q, R$ and $[Q, R]$ are in class (a), (b) or (c), then $\operatorname{TR}[Q, R]=0$. 
It is possible to calculate $\operatorname{KERD}(Q, x)$ from $K(Q, x, y)$ without making an analytic continuation. In the case when $d$ is nonintegral this was shown in $[\mathrm{KV}]$. Here we will show how to do it in the more delicate case when $Q$ is odd class. We make a splitting $Q=Q_{\text {reg }}+Q_{\text {sing }}$ with $Q_{\text {reg }}$ smoothing, so that the following result holds.

THEOREM 1.2.2.

$$
\operatorname{KERD}(Q, x)=K\left(Q_{\mathrm{reg}}, x, x\right), \quad \operatorname{TR} Q=\operatorname{TR} Q_{\text {reg }}
$$

If $\partial$ is a differential operator on $E$ then

$$
\operatorname{KERD}(\partial Q, x)=\left.\partial_{x} K\left(Q_{\mathrm{reg}}, x, y\right)\right|_{y=x}, \quad \operatorname{TR} \partial Q=\operatorname{TR} \partial Q_{\mathrm{reg}} .
$$

The splitting $Q=Q_{\text {reg }}+Q_{\text {sing }}$ is made by examining the asymptotic behavior of the Schwartz kernel $K(Q, x, y)$ of $Q$ close to the diagonal, and separating the regular terms from the singular terms. The operator $Q_{\text {sing }}$ is what we call purely singular, a concept which we now define.

Definition 1.2.3. (a) Let $q(\xi)$ be a tempered distribution on $\mathbb{R}^{n}$ which is homogeneous of degree $j$. Then $q$ has regular parity if it satisfies (1.2.3) on $\mathbb{R}^{n}$ in the distributional sense, and has singular parity if it satisfies (1.2.4) in the distributional sense.

(b) Suppose $S$ and $S_{j}$ are $\mathbb{C}(N)$-valued tempered distributions on $\mathbb{R}^{n}$ for $j=d, d+1, \ldots$, and suppose $S_{j}$ is homogeneous of degree $j$ on $\mathbb{R}^{n}$ and smooth away from the origin. We write

$$
S(w) \sim S_{d}(w)+S_{d+1}(w)+\ldots, \quad w \rightarrow 0,
$$

if

$$
S(w)-S_{d}(w)-\cdots-S_{j}(w)\left\{\begin{array}{l}
\in C^{j}\left(\mathbb{R}^{n}\right) \\
=O\left(|w|^{j+1}\right), \quad w \rightarrow 0 .
\end{array}\right.
$$

(c) If $S$ satisfies (1.2.8), we say that $S$ has regular parity, respectively singular parity, in $w$ as $w \rightarrow 0$ if every $S_{j}$ has regular parity, respectively singular parity in $w$.

(d) When $n$ is odd, the odd class pseudodifferential operator $Q$ is purely singular if the Schwartz kernel in local coordinates has the form $K(Q, x, y)=$ $S(x, x-y)$ where $S(x, w)$ has singular parity in $w$ as $w \rightarrow 0$. In particular differential operators have singular parity.

It is easy to show that the Schwartz kernel of an odd class operator $Q$ can be split in local coordinates as

$$
K(Q, x, y)=R(x, x-y)+S(x, x-y)
$$


where $R$ is smooth and $S(x, w)$ has singular parity as $w \rightarrow 0$. The operator $Q_{\text {sing }}$ is formed by patching together the kernels $S(x, x-y)$ corresponding to different coordinate charts, and the result is a purely singular operator. It will be shown that the canonical trace of any purely singular operator vanishes, which leads to Theorem 1.2.2. The details of this argument are given in Section 2.2 .

Example 1.2.4. For the Laplacian on $S^{3}$, the eigenvalues are $k(k+2)$ with multiplicity $(k+1)^{2}$. Explicit calculation gives

$$
\operatorname{TR} \Delta^{-1}=Z(1)=\left.\sum_{k=1}^{\infty} \frac{(k+1)^{2}}{(k(k+2))^{s}}\right|_{s=1} ^{\text {mer }}=-\frac{3}{4} .
$$

On the other hand writing $d \mu_{3}$ for surface measure on $S^{3}$ normalized to total volume 1 , and $r$ for the distance between points $x$ and $y$ on $S^{3}$, the Schwartz kernel $K\left(\Delta^{-1}, x, y\right)$ has the form $G(r) d \mu_{3}(y)$ where $G$ satisfies the differential equation

$$
\frac{1}{\sin ^{2} r} \frac{d}{d r} \sin ^{2} r \frac{d}{d r} G(r)=1, \quad \int_{0}^{\pi} G(r) \sin ^{2} r d r=0 .
$$

The solution is

$$
G(r)=\frac{(\pi-r) \cos r}{2 \sin r}-\frac{1}{4}
$$

The regular part and singular parts for $r$ small are given by

$$
G_{\text {reg }}(r)=-\frac{r \cos r}{2 \sin r}-\frac{1}{4}, \quad G_{\operatorname{sing}}(r)=\frac{\pi \cos r}{2 \sin r},
$$

and so by Theorem 1.2.2,

$$
\operatorname{TR} \Delta^{-1}=\int_{S^{3}} G_{\mathrm{reg}}(0) d \mu_{3}=G_{\mathrm{reg}}(0)=-\frac{3}{4} .
$$

We see that these two calculations agree.

Next we state a small technical lemma which we need in order to describe our second result on computing the canonical trace.

Lemma 1.2.5. (a) If $n$ is odd and $q(\xi) \in C^{\infty}\left(\mathbb{R}^{n} \backslash 0\right)$ is a smooth homogeneous function on $\mathbb{R}^{n} \backslash 0$ of degree $d \in \mathbb{Z}$ and regular parity, then there is a unique homogeneous distribution with regular parity on $\mathbb{R}^{n}$ which equals $q$ on $\mathbb{R}^{n} \backslash 0$.

An important consequence of this lemma is that it gives a canonical way to multiply together the kernels of two odd class operators.

For an integrable function $q$, define the Fourier and inverse Fourier transforms of $q$ by

$$
\hat{q}(w)=\int_{\mathbb{R}^{n}} q(\xi) e^{-i w \cdot \xi} d \xi, \quad \check{q}(w)=\frac{1}{(2 \pi)^{n}} \int_{\mathbb{R}^{n}} q(\xi) e^{i w \cdot \xi} d \xi=\frac{1}{(2 \pi)^{n}} \hat{q}(-w) .
$$


This definition extends to tempered distributions on $\mathbb{R}^{n}$. For a symbol $q(x, \xi)$, define $\hat{q}(x, w)$ and $\breve{q}(x, w)$ to be the Fourier and inverse Fourier transforms respectively in the second variable. We state the following theorem for vector bundles since we will need this generality in applications. However, it can be easily deduced from the scalar case.

Theorem 1.2.6. (a) Suppose that $E_{i} \rightarrow M, i=0,1,2,3$ are vector bundles over $M$, that $A \in C^{\infty}\left(E_{2} \otimes E_{1}^{*}\right)$ and $Q: C^{\infty}\left(E_{0}\right) \rightarrow C^{\infty}\left(E_{1}\right)$, $P: C^{\infty}\left(E_{2}\right) \rightarrow C^{\infty}\left(E_{3}\right)$ are odd class pseudodifferential operators. Then $S: C^{\infty}\left(E_{2} \otimes E_{1}^{*}\right) \rightarrow C^{\infty}\left(E_{3} \otimes E_{0}^{*} \otimes \wedge\right)$ defined by

$$
S A(x)=\operatorname{KERD}(P A Q, x)
$$

is a classical pseudodifferential operator whose Schwartz kernel is

$$
K(P, x, y) \otimes K(Q, y, x) .
$$

(b) If $\operatorname{ord} P=\alpha$ and $\operatorname{ord} Q=\beta$ and $\alpha, \beta>-n$, and the principal symbols of $P$ and $Q$ are $p_{\alpha}$ and $q_{\beta}$ respectively, then the principal symbol of $S$ is

$$
s(x, \xi)=\left(\check{p}_{\alpha} \otimes \check{q}_{\beta}^{\prime}\right)^{\wedge}(x, \xi) \in \pi^{*}\left(E_{3} \otimes E_{2}^{*} \otimes E_{1} \otimes E_{0}^{*}\right),
$$

where $q_{\beta}^{\prime}(x, \xi)=q_{\beta}(x,-\xi)$ and $\pi: T^{*} M \rightarrow M$ is the natural projection.

Remarks. 1. The definition of odd class operators acting between two different vector bundles over $M$ is identical to that given in Definition 1.2.1 for operators acting on a single vector bundle.

2. To define the tensor product of distributions $K(P, x, y) \otimes K(Q, y, x)$, decompose $P=P_{\text {reg }}+P_{\text {sing }}, Q=Q_{\text {reg }}+Q_{\text {sing }}$. Now the products

$$
\begin{aligned}
& K\left(P_{\mathrm{reg}}, x, y\right) \otimes K\left(Q_{\mathrm{reg}}, y, x\right), \\
& K\left(P_{\mathrm{reg}}, x, y\right) \otimes K\left(Q_{\mathrm{sing}}, y, x\right), \\
& K\left(P_{\mathrm{sing}}, x, y\right) \otimes K\left(Q_{\mathrm{reg}}, y, x\right)
\end{aligned}
$$

are well-defined, being products of distributions with smooth functions. The problematic term is

$$
K\left(P_{\text {sing }}, x, y\right) \otimes K\left(Q_{\text {sing }}, y, x\right) .
$$

This is well-defined as a smooth function away from the diagonal, which in local coordinates has regular parity in $x-y$ as $y \rightarrow x$. By Lemma 1.2.5, this extends uniquely over the diagonal to a regular parity distribution, which we take as the definition of the product in 1.2.11. More explicitly, there is a polyhomogeneous expansion in local coordinates,

$$
\begin{aligned}
& K\left(P_{\text {sing }}, x, y\right)=S(x, x-y), \quad S(x, w) \sim S_{\alpha}(x, w)+S_{\alpha+1}(x, w)+\ldots, \\
& K\left(Q_{\text {sing }}, y, x\right)=T(x, x-y), \quad T(x, w) \sim T_{\beta}(x, w)+T_{\beta+1}(x, w)+\ldots,
\end{aligned}
$$


which leads to an expansion for the product of the functions $S(x, w)$ and $T(x, w)$ away from $w=0$,

$S(x, w) \otimes T(x, w) \sim P_{\alpha+\beta}(x, w)+P_{\alpha+\beta+1}(x, w)+\ldots, \quad P_{j}=\sum_{k+\ell=j} S_{k} \otimes T_{\ell}$,

so that $P_{j}(x, w)$ is homogeneous of degree $j$ in $w$. The difference

$$
S(x, w) \otimes T(x, w)-\sum_{j \leq-n} P_{j}(x, w)
$$

is an integrable function and each of the terms $P_{j}(x, w)$ is homogeneous of regular parity and hence extends to a regular parity distribution in $w$ by Lemma 1.2.5. The fact that this extension is independent of coordinates follows from the fact that the property of being a regular or singular parity distribution is invariant under a change of coordinates, which is proved in Section 2.2.

In Section 5.1, we present some additional results concerning the calculation of the canonical trace for certain operators on spheres.

1.3. Critical metrics. We begin with a result describing the behavior of $\operatorname{det}^{\prime} \Delta$ and $\operatorname{det} L$ in the neighborhood of a general critical metric when the dimension $n$ is odd. The result states roughly that every critical metric has finite index.

Write $\mathcal{M}$ for the space of metrics on $M$. The tangent space $T_{g_{0}} \mathcal{M}$ at the metric $g_{0}$ is the space $C^{\infty}\left(S^{2} T^{*} M\right)$ of smooth symmetric $(0,2)$ tensor fields on $M$. Some basic theory of the space $\mathcal{M}$ can be found in [Eb].

For the most part, we will work in a frame which is orthonormal with respect to a fixed metric $g_{0}$. At each point $x \in M$, take an orthonormal basis $e_{1}, \ldots, e_{n}$ for the tangent space $T_{x} M$. Unless otherwise stated, the components of tensor fields are given with respect to this basis, with all indices written as subscripts. Covariant derivatives in the directions $e_{i}, e_{j}$ etc. will be denoted by $D_{i}, D_{j}$ etc. or by the use of subscripts. For example, if $A \in T_{g_{0}} \mathcal{M}$, then

$$
A_{i j, k \ell}=D_{\ell} D_{k} A_{i j}=\left(D^{2} A\right)\left(e_{i}, e_{j}, e_{k}, e_{\ell}\right) .
$$

In this notation, the metric $g_{0}$ is the $(0,2)$ tensor field $\delta_{i j}$. We use the summation convention that any repeated index is summed over. Write $d \mu_{0}$ for the normalized canonical volume element for the metric $g_{0}$, with normalization $\mu_{0}(M)=1$. The space $T_{g_{0}} \mathcal{M}$ has a scalar product

$$
\langle a, b\rangle=\int_{M} a_{i j} b_{i j} d \mu_{0} .
$$

The group of diffeomorphisms of $M$ acts on $\mathcal{M}$ in a natural way; if $\psi: M \rightarrow M$ is a diffeomorphism then $\psi\left(g_{0}\right)$ is the pull-back $\left(\psi^{-1}\right)^{*} g_{0}$. We denote the orbit of $g_{0}$ by $\operatorname{Diff}\left(g_{0}\right)$. Then $\operatorname{det}^{\prime} \Delta$ is constant on this orbit because 
every metric $g \in \operatorname{Diff}\left(g_{0}\right)$ is isometric to $g_{0}$ and hence isospectral to $g_{0}$. Write $\operatorname{diff}\left(g_{0}\right)$ for the tangent space of $\operatorname{Diff}\left(g_{0}\right)$ at $g_{0}$. This space contains tensor fields on $M$ of the form $X_{i, j}+X_{j, i}$ where $X$ is any 1-form. The perpendicular space $\operatorname{diff}\left(g_{0}\right)^{\perp}$ contains divergence-free fields, that is, fields of the form $A_{i j}$ with $A_{i j, j}=0$. Write $\operatorname{Conf}\left(g_{0}\right)$ for the space of metrics conformal to $g_{0}$. As we have already remarked, in odd dimensions $\operatorname{det} L$ is constant on $\operatorname{Conf}\left(g_{0}\right)$. Let $\operatorname{conf}\left(g_{0}\right)$ be the tangent space to $\operatorname{Conf}\left(g_{0}\right)$ at $g_{0}$, which contains tensor fields on $M$ of the form $\phi \delta_{i j}$ where $\phi$ is a smooth function on $M$. It can be proved using elliptic regularity theory that we have splittings

$$
\begin{aligned}
& T_{g_{0}} \mathcal{M}=\operatorname{diff}\left(g_{0}\right) \oplus \operatorname{diff}\left(g_{0}\right)^{\perp}, \\
& T_{g_{0}} \mathcal{M}=\operatorname{diff}\left(g_{0}\right)+\operatorname{conf}\left(g_{0}\right) \oplus\left(\operatorname{diff}\left(g_{0}\right)+\operatorname{conf}\left(g_{0}\right)\right)^{\perp},
\end{aligned}
$$

where for a subspace $V$ of $T_{g_{0}} \mathcal{M}, V^{\perp}$ is the orthogonal complement of $V$ in $T_{g_{0}} \mathcal{M}$. Since $\operatorname{det}^{\prime} \Delta$ scales like $V^{2 / n}$ where $V$ is the volume, it is computationally simpler to work with the functional

$$
F=\log \operatorname{det}^{\prime} \Delta-\frac{2}{n} \log V
$$

which is homogeneous of degree zero under scaling. The metric $g_{0}$ is critical or maximal for $\operatorname{det}^{\prime} \Delta$ under deformations which preserve the volume if and only if it is critical, respectively maximal for $F$ under all deformations.

TheOREm 1.3.1. Suppose the dimension $n \geq 3$ of $M$ is odd and let $g_{0}$ be critical for $F$. Then

$$
\text { Hess } F(X, Y)=0, \quad \text { for all } X \in \operatorname{diff}\left(g_{0}\right), Y \in T_{g_{0}} \mathcal{M} \text {. }
$$

Moreover, Hess $F$ restricted to the subspace $\left(\operatorname{diff}\left(g_{0}\right)\right)^{\perp}$ of $T_{g_{0}} \mathcal{M}$ has at most finitely many nonpositive eigenvalues if $n=1 \bmod 4$ and at most finitely many nonnegative eigenvalues if $n=3 \bmod 4$. Similarly, if $g_{0}$ is critical for $\log |\operatorname{det} L|$, then

Hess $\log |\operatorname{det} L|(X, Y)=0, \quad$ for all $X \in \operatorname{diff}\left(g_{0}\right)+\operatorname{conf}\left(g_{0}\right), Y \in T_{g_{0}} \mathcal{M}$.

Moreover, Hess $\log |\operatorname{det} L|$ restricted to the subspace $\left(\operatorname{diff}\left(g_{0}\right)+\operatorname{conf}\left(g_{0}\right)\right)^{\perp}$ has at most finitely many nonpositive eigenvalues if $n=1 \bmod 4$ and at most finitely many nonnegative eigenvalues if $n=3 \bmod 4$.

This result has the following immediate corollary:

COROllary 1.3.2. If $n=3 \bmod 4$, then no metric can be a local minimum for $\operatorname{det}^{\prime} \Delta$ under deformations fixing the volume. If $n=1 \bmod 4$, then no metric can be a local maximum for $\operatorname{det}^{\prime} \Delta$ under deformations fixing the volume. The same is true for the functional $|\operatorname{det} L|$ where it does not vanish. 
Theorem 1.3.1 also indicates that the set of critical Riemannian manifolds for $\operatorname{det}^{\prime} \Delta$ or $\operatorname{det} L$ is finite dimensional. We calculate explicitly what sort of critical point the standard sphere is and the results are summarized in the following table.

THEOREM 1.3.3. When $n$ is odd, the standard $n$-sphere is critical for $\operatorname{det} L$ under all smooth deformations, and critical for $\operatorname{det}^{\prime} \Delta$ under deformations fixing the volume. We describe the types of critical points in several cases in the following table.

\begin{tabular}{|c|c|c|c|}
\hline standard sphere & extremal type & for & among metrics fixing \\
\hline$S^{3}$ & local max & $\operatorname{det}^{\prime} \Delta$ & volume + smooth structure* \\
$S^{4 m+3}, m=1,2, \ldots$ & saddle & $\operatorname{det}^{\prime} \Delta$ & volume + conformal class \\
$S^{4 m+3}$ & local max & $\operatorname{det} L$ & smooth structure ** \\
$S^{4 m+1}$ & local min & $\operatorname{det} L$ & smooth structure** \\
\hline
\end{tabular}

*extends Richardson's result outside the conformal class,

**only nontrivial across conformal classes.

In particular, notice that the standard 3 -sphere is a local maximum for $\operatorname{det}^{\prime} \Delta$, while the standard $4 m+3$-sphere is a saddle for $m=1,2, \ldots$ There is overwhelming evidence that for all odd $n>5$, the $n$-sphere is a saddle point for $\operatorname{det}^{\prime} \Delta$ under conformal deformations preserving the volume. We prove the case $n=4 m+3$ in Section 6 . The case $n=4 m+1$ is computationally more complicated, but we have checked $n=9,13,17$ with the help of Mathematica. In fact there is a clear pattern to how the Hessian of the determinant of the Laplacian behaves for conformal deformations, and this is described at the end of this section.

The local extrema in the above table are all strict in the sense of the following results.

THEOREM 1.3.4. Let $g_{0}$ be the standard metric on $S^{3}$ and let $g(t)$ be a deformation of $g_{0}$. If $g(t)$ fixes the volume and $\dot{g}(0) \notin \operatorname{diff}\left(g_{0}\right)$, then $\operatorname{det}^{\prime} \Delta_{g(t)}$ has a strict local maximum at $t=0$.

THEOREM 1.3.5. Let $g_{0}$ be the standard metric on $S^{n}$ where $n$ is odd and $n \geq 3$, and let $g(t)$ be a deformation of $g_{0}$. If $\dot{g}(0) \notin \operatorname{diff}\left(g_{0}\right)+\operatorname{conf}\left(g_{0}\right)$ then $\operatorname{det} L_{g(t)}$ has a strict local maximum at $t=0$ if $n=3 \bmod 4$, and a strict local minimum if $n=1 \bmod 4$. 
We will prove Theorems 1.3.4 and 1.3.5 in this paper. From Theorem 1.3.4, it follows by applying the Nash-Moser implicit function theorem that if $g(t)$ is a deformation of the standard metric $g_{0}$ on $S^{3}$ with $\dot{g}(0) \in \operatorname{diff}\left(g_{0}\right)$, then $\operatorname{det}^{\prime} \Delta_{g(t)}$ has a local maximum at $t=0$. The details will be given in a subsequent paper [Ok4], where upper bounds on $\operatorname{det}^{\prime} \Delta$ for metrics close to $S^{3}$ will also be given, as well as the analogous results for $\operatorname{det} L$ close to standard spheres.

Our next results characterize the critical metrics for $\operatorname{det} L$ and $\operatorname{det}^{\prime} \Delta$. Let $\mu_{0}$ be the canonical volume element for $g_{0}$ normalized so that $\mu_{0}(M)=1$. Let $Q$ be $\Delta_{0}$ or $L_{0}$ and let $G(x, y)$ be Green's function for $Q$; that is, $G(x, y) d \mu_{0}(y)$ is the Schwartz kernel for $Q^{-1}$. Then $G(x, y)$ is a smooth function on $M \times M$ except at the diagonal where it blows up. Define $\mathcal{G}(x, y)$ to be the regular part of the Green function; that is, $\mathcal{G}(x, y) d \mu_{0}(y)$ is the Schwartz kernel for

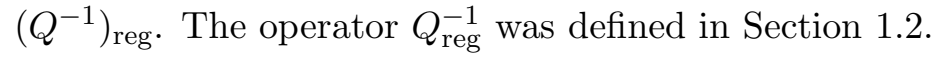

THEOREM 1.3.6 (first variation formula for $F$ ). Let $g_{0}$ be a metric on $M$ which has odd dimension $n \geq 3$. Write $\mathcal{G}(x, y)$ for the regular part of the Green function for $\Delta_{0}$. Let $g(t)$ be a deformation of $g_{0}$, and write $\dot{g}(0)=2 \phi \delta_{i j}+A_{i j}$ where $A_{i i}=0$. Then

$$
\left.\frac{d F(g(t))}{d t}\right|_{t=0}=\int\left((n-2) \phi \delta_{i j}-A_{i j}\right)\left(\left.D_{x, j} D_{y, i} \mathcal{G}(x, y)\right|_{y=x}+\frac{1}{n} \delta_{i j}\right) d \mu .
$$

Remark. The notation $\left.D_{x, i} D_{y, j} \mathcal{G}(x, y)\right|_{y=x}$ should be interpreted in the following sense. The tangent and cotangent spaces of $M \times M$ split canonically as products and this enables us to take covariant derivatives in each variable separately in a canonical way. (In fact the covariant derivatives $D_{x, i}$ and $D_{y, j}$ commute with each other.) The $(0,2)$ tensor field $D_{x, i} D_{y, j} G(x, y)$ on $M \times M$ can be pulled back to $M$ by the diagonal map $x \rightarrow(x, x)$.

Theorem 1.3.6 leads to the following characterization of critical metrics for $F$.

Corollary 1.3.7. When the dimension $n \geq 3$ of $M$ is odd, the following conditions (1.3.1), (1.3.2), (1.3.3) and (1.3.4) are equivalent:

The metric $g_{0}$ is critical for $\operatorname{det}^{\prime} \Delta$ under all deformations of $g_{0}$ which fix the volume.

$$
\left.D_{y, i} D_{x, j} \mathcal{G}(x, y)\right|_{y=x}=-\frac{\delta_{i j}}{n}
$$


(a) $\mathcal{G}(x, x)$ is independent of $x$ and

$$
\left.\left(D_{x, i} D_{x, j} \mathcal{G}(x, y)\right)\right|_{y=x}=\frac{\delta_{i j}}{n}
$$

$$
\text { (a) } \mathcal{G}(x, x) \text { is independent of } x
$$

and

(b) $\left.\quad\left(D_{x, i} D_{x, j} \mathcal{G}(x, y)\right)\right|_{y=x}=c \delta_{i j}, \quad c$ constant.

Here, $\mathcal{G}(x, y)$ is the regular part of the Green function of $\Delta_{g_{0}}$.

Remark. The condition (1.3.4)(a) holds if and only if $g_{0}$ is critical for $\operatorname{det}^{\prime} \Delta$ under conformal deformations which fix the volume. This was proved by K. Richardson in [Ri]. The constant $\mathcal{G}(x, x)$ equals $Z(1)$ where $Z(s)$ is the zeta function for $\Delta_{0}$.

We have similar results for the conformal Laplacian.

TheOREM 1.3.8. Suppose that the dimension $n \geq 3$ of $M$ is odd. Write $G(x, y)$ for Green's function for $L$, and $\mathcal{G}(x, y)$ for the regular part of $G(x, y)$. Write $R_{i j}$ for the Ricci curvature tensor. Let $g_{0}$ be a metric on $M$ for which $L_{g_{0}}$ is invertible and let $g(t)$ be a deformation of $g_{0}$. Decompose $\dot{g}_{i j}(0)=2 \phi \delta_{i j}+A_{i j}$ where $A_{i i}=0$. Now,

$$
\begin{aligned}
\frac{d \log \operatorname{det} L}{d t} & =\int \dot{g}_{i j}(0)\left(\left.\left(D_{x, j} D_{x, i}-\alpha R_{i j}\right) \mathcal{G}(x, y)\right|_{y=x}\right. \\
\left.-\frac{n}{4(n-1)}\left(D_{i} D_{j}+\frac{\delta_{i j}}{n} \Delta\right) \mathcal{G}(x, x)\right) d \mu_{0} & \\
= & \left.-\frac{n}{4(n-1)} A_{i j}(0)\left(\left.\left(D_{x, j} D_{x, i}-\alpha R_{i j}\right) \mathcal{G}(x, y)\right|_{y=x}(x, x)\right)\right) d \mu_{0}
\end{aligned}
$$

This leads to the following characterization of critical metrics for $\log \operatorname{det} L$.

Corollary 1.3.9. Suppose that the dimension $n \geq 3$ of $M$ is odd. The following conditions (1.3.5), (1.3.6) and (1.3.7) are equivalent.

The metric $g_{0}$ is critical for $\operatorname{det} L$ under all deformations of $g_{0}$. 


$$
\begin{gathered}
\left.\left(-D_{x, i} D_{x, j}+\frac{n-2}{4(n-1)} R_{i j}(x)\right) \mathcal{G}(x, y)\right|_{y=x} \\
+\frac{n}{4(n-1)} D_{i} D_{j}(\mathcal{G}(x, x))=c_{0} \delta_{i j} \quad \text { with } c_{0} \text { constant } \\
\left.\left(-D_{x, i} D_{x, j}+\frac{n-2}{4(n-1)} R_{i j}(x)\right) \mathcal{G}(x, y)\right|_{y=x} \\
=-\frac{1}{4(n-1)}\left(n D_{i} D_{j}+\delta_{i j} \Delta_{0}\right)(\mathcal{G}(x, x)),
\end{gathered}
$$

where $R_{i j}$ is the Ricci curvature tensor and $\mathcal{G}(x, y)$ is the regular part of the Green function of $L_{g_{0}}$.

Remark. It is clear that the standard metric on $S^{n}$ satisfies the conditions (1.3.4) and (1.3.6), and hence is critical for $\operatorname{det}^{\prime} \Delta$ and $\operatorname{det} L$.

To prove Theorem 1.3.8, we have

$$
\frac{d}{d t} \log \operatorname{det} L=\operatorname{TR} \dot{L} L^{-1}=\left.\int_{M} \dot{L}_{x} \mathcal{G}(x, y)\right|_{y=x} d x
$$

where $\mathcal{G}$ is the regular part of the Green function for $L$ and the first equality is established in $[\mathrm{KV}]$ and the second equality follows from Theorem 1.2.2. Computing the right-hand side of (1.3.8) gives Theorem 1.3.8. A similar calculation holds for the ordinary Laplacian and all the details are carried out in Sections 2.1 and 2.2 .

Now we consider the second variation of the functionals $F$ and $\log \operatorname{det} L$. We have

$$
\frac{d^{2}}{d t^{2}} \log \operatorname{det} L=\frac{d}{d t} \operatorname{TR} \dot{L} L^{-1}=\operatorname{TR} \ddot{L} L^{-1}+\operatorname{TR} \dot{L} L^{-1} \dot{L} L^{-1}
$$

By Theorem 1.2.2,

$$
\operatorname{TR} \ddot{L} L^{-1}=\left.\int_{M} \ddot{L}_{x} \mathcal{G}(x, y)\right|_{y=x} d x
$$

The term TR $\dot{L} L^{-1} \dot{L} L^{-1}$ can be somewhat simplified. The functional $F$ can be treated similarly, leading to the following second variation formulas.

Theorem 1.3.10. Suppose $\phi \in C^{\infty}(M)$, let $g_{0}$ be a critical metric for $F$ and let $A$ be a symmetric (0,2)-tensor field on $M$ which is pointwise trace-free with respect to $g_{0}$; that is, $A_{i i}=0$. Write

$$
\bar{\phi}=\phi-\int_{M} \phi d \mu_{0}, \quad \bar{\Delta}_{0}^{-1}=\Delta_{0}^{-1}-Z(1) \Pi_{0},
$$


where $Z(s)$ is the zeta function for $\Delta_{g_{0}}$. Then

$$
\begin{gathered}
\text { Hess } F\left(2 \phi g_{0}+A, 2 \phi g_{0}+A\right)=-\frac{1}{n} \int_{M} A_{i j} A_{i j} d \mu_{0}+\frac{(n+2)(n-2)}{2} \int_{M} \bar{\phi}^{2} d \mu_{0} \\
-\operatorname{TR}\left(\frac{n-2}{2} \phi_{i i}+D_{i} A_{i j} D_{j}\right) \bar{\Delta}_{0}^{-1}\left(\frac{n-2}{2} \phi_{k k}+D_{k} A_{k \ell} D_{\ell}\right) \bar{\Delta}_{0}^{-1} .
\end{gathered}
$$

Theorem 1.3.11. Suppose $g_{0}$ is critical for $\log \operatorname{det} L$ and suppose $A \in$ $T_{g_{0}} \mathcal{M}$ has $A_{i i}=0$ and $A_{i j, j}=0$. Then

$$
\begin{aligned}
\text { Hess log } \operatorname{det} L(A, A)= & \frac{1}{4(n-1)} \operatorname{TR}\left(A_{i j} A_{i j}\right)_{k k} L^{-1} \\
& +\alpha \operatorname{TR}\left(A_{i j, k} A_{i k, j}-\frac{1}{2} A_{i j, k} A_{i j, k}\right) L^{-1} \\
& -\operatorname{TR} A_{i j}\left(D_{i} D_{j}-\alpha R_{i j}\right) L^{-1} A_{k \ell}\left(D_{k} D_{\ell}-\alpha R_{k \ell}\right) L^{-1} .
\end{aligned}
$$

Explicit evaluation of (1.3.10) at the standard 3-sphere leads to Theorem 1.3.4. Explicit evaluation of (1.3.11) at the standard $n$-sphere with $n$ odd leads to Theorem 1.3.5. We now explain in more detail what happens to the functional $F$ close to the standard $n$-sphere for odd $n>3$.

Let $g_{0}$ denote the standard metric on $S^{n}$. From Theorem 1.3.10,

$$
\begin{aligned}
\operatorname{Hess} F\left(2 \phi g_{0}, 2 \phi g_{0}\right)= & \frac{(n+2)(n-2)}{2} \int_{S^{n}} \bar{\phi}^{2} d \mu_{n} \\
& -\frac{(n-2)^{2}}{4} \operatorname{TR} \phi_{i i} \bar{\Delta}_{0}^{-1} \phi_{k k} \bar{\Delta}_{0}^{-1} .
\end{aligned}
$$

From Theorem 1.2.6,

$$
\operatorname{TR} \phi_{i i} \bar{\Delta}_{0}^{-1} \phi_{k k} \bar{\Delta}_{0}^{-1}=\int_{S^{n}} \phi_{i i} T \phi_{k k} d \mu_{n}
$$

where $T$ is a pseudodifferential operator with

$$
K(T, x, y)=K\left(\bar{\Delta}_{0}^{-1}, x, y\right) K\left(\bar{\Delta}_{0}^{-1}, y, x\right) .
$$

This kernel is invariant under transformations of $S^{n} \times S^{n}$ which preserve the distance $r$ between $x$ and $y$ and hence $T$ is a function of the Laplacian, and

$$
\operatorname{Hess} F\left(2 \phi g_{0}, 2 \phi g_{0}\right)=\int_{S^{n}} \phi Q \phi d \mu_{n}
$$

where $Q$ is a pseudodifferential operator which is a function of the Laplacian.

Write $\mathcal{H}_{k}$ for the space of spherical harmonics of degree $k$ on $S^{n}$. This is the space of homogeneous harmonic, degree $k$ polynomials on $\mathbb{R}^{n+1}$ restricted to $S^{n}$, and is an eigenspace for the Laplacian on $S^{n}$ and hence an eigenspace 
for $Q . \mathcal{H}_{0}$ is the space of constants, and $Q$ vanishes on $\mathcal{H}_{0}$ since $F$ is scale invariant. $\mathcal{H}_{1}$ is the space of linear functions on $\mathbb{R}^{n}$ restricted to $S^{n}$ and equals $\operatorname{conf}\left(g_{0}\right) \cap \operatorname{diff}\left(g_{0}\right)$, so $Q$ vanishes on $\mathcal{H}_{1}$. By Theorem 1.3.1, we see that when $n=3 \bmod 4, Q$ is strictly negative on a space of finite codimension; so for $k$ sufficiently large, $Q$ is strictly negative on $\mathcal{H}_{k}$.

Lemma 1.3.12. If $n>3$ is odd and $\phi \in \mathcal{H}_{2}$ then

$$
\operatorname{Hess} F\left(2 \phi g_{0}, 2 \phi g_{0}\right)=\frac{2(n-2)\left(-2+(n-2)(n+1) \sum_{j=2}^{n-1} \frac{1}{j}\right)}{(n-3) n} \int_{S^{n}} \phi^{2} d \mu_{n} \text {. }
$$

Since the right-hand side is clearly positive for $n>3$, we see that if $n=3$ $\bmod 4$, then $Q$ has both positive and negative eigenvalues and $S^{n}$ is a saddle point for $F$. Concerning the case $n=1 \bmod 4$, computer calculations indicate that $S^{5}$ is a local minimum for $F$ among metrics in the same conformal class, while $S^{n}$ is a saddle point for all odd $n>5$. Indeed, it appears that the index of this saddle point has a simple description, since for low values of $k$, the sign of Hess $F$ on $\mathcal{H}_{k}$ appears to have a 4 -fold periodicity in $k$.

Conjecture 1.3.13.

$$
\begin{aligned}
1<k<n-1 & \Rightarrow \begin{cases}\text { Hess } F<0 \text { on } \mathcal{H}_{k} & \text { if } k=1 \quad \bmod 4, \\
\operatorname{Hess} F>0 \text { on } \mathcal{H}_{k} & \text { if } k=2,3,0 \bmod 4,\end{cases} \\
k \geq n-1 & \Rightarrow \begin{cases}\text { Hess } F<0 \text { on } \mathcal{H}_{k} & \text { if } n=3 \bmod 4, \\
\operatorname{Hess} F>0 \text { on } \mathcal{H}_{k} & \text { if } n=1 \quad \bmod 4 .\end{cases}
\end{aligned}
$$

This conjecture is confirmed by computer calculations using Mathematica for the case that $n$ is odd, $3 \leq n \leq 17$ and $k \leq 20$.

The outline of the paper is as follows. Section 2 contains the proofs of the results on the canonical trace described in Section 1.2, including proofs of Theorems 1.2.2 and 1.2.6.

In Sections 3.1 and 3.2 we compute first variation formulas for $F$ and $\log \operatorname{det} L$ and characterize the critical metrics; that is, we prove Theorems 1.3.6-1.3.9. In Sections 3.3 and 3.4 we compute the second variation formulas for $F$ and $\log \operatorname{det} L$ given in (1.3.10) and (1.3.11).

In Section 4 we apply Theorem 1.2.6 to analyze those terms in (1.3.10) and (1.3.11) which involve the canonical trace. This leads to the proof that critical points of $\operatorname{det}^{\prime} \Delta$ and $\operatorname{det} L$ have finite index as described in Theorem 1.3.1. 
In Section 5 we compute (1.3.10) and (1.3.11) explicitly at the standard spheres in certain dimensions to verify the extremal properties of Theorems 1.3.3 and 1.3.4, while in Section 6, we compute (1.3.10) explicitly at the standard spheres for conformal deformations corresponding to spherical harmonics in $\mathcal{H}_{2}$, to prove Lemma 1.3.12.

Theorem 1.3.1 and the 3-dimensional case of Theorem 1.3.3 were circulated in a preprint in 1997. The proofs appearing in this paper are slightly simpler than the original ones. In a sequel to this paper [Ok3], we will describe the behavior of critical metrics for special values of the zeta function. We have observed a marked difference in behavior between $Z(k)$ when it is summable, and when it is not.

I would like to thank Peter Sarnak for suggesting that I investigate the determinant of the Laplacian on 3-manifolds. I would also like to thank Alice Chang, Richard Hamilton, David Jerison, Ken Richardson, Gang Tian, Nolan Wallach and Paul Yang for useful conversations.

\section{Regularization theorems}

In this section we prove the results stated and outlined in Section 1.2.

2.1. Symbols. For $\Omega \subset \mathbb{R}^{n}$, write $\mathcal{D}(\Omega)$ for the space of smooth compactly supported functions on $\Omega$ and $\mathcal{D}^{\prime}(\Omega)$ for the space of distributions on $\Omega$.

Proof of Lemma 1.2.5. Consider the function $q(\xi)|\xi|^{z}$. This is homogeneous in $\xi$ of degree $z+d$ and hence when $\Re z+d>-n$ it is locally integrable

and gives a homogeneous distribution $q_{z}(\xi)$ of degree $z+d$. For $f$ a Schwartz function, set

$$
f_{N}(\xi)=f(\xi)-\sum_{|\alpha|<N} \frac{\partial_{\xi}^{\alpha} f(0)}{\alpha !} \xi^{\alpha},
$$

so that $f_{N}(\xi)=O\left(|\xi|^{N}\right)$. Then $\left\langle q_{z}(\xi), f(\xi)\right\rangle$ equals

$$
\begin{aligned}
\int_{|\xi|>1} q(\xi)|\xi|^{z} f(\xi) d \xi & \\
+ & \int_{|\xi|<1} q(\xi)|\xi|^{z} f_{N}(\xi) d \xi+\int_{|\xi|<1} q(\xi)|\xi|^{z} \sum_{|\alpha|<N} \frac{\partial_{\xi}^{\alpha} f(0)}{\alpha !} \xi^{\alpha} d \xi \\
=\int_{|\xi|>1} q(\xi)|\xi|^{z} f(\xi) d \xi & +\int_{|\xi|<1} q(\xi)|\xi|^{z} f_{N}(\xi) d \xi \\
& +\sum_{|\alpha|<N} \frac{\partial_{\xi}^{\alpha} f(0)}{\alpha !(z+d+|\alpha|+n)} \int_{|\theta|=1} q(\theta) \theta^{\alpha} d \sigma_{n-1}(\theta)
\end{aligned}
$$


where $d \sigma_{n-1}$ is surface measure on the unit sphere in $\mathbb{R}^{n}$. Since the final expression defines a meromorphic function of $z$ when $\Re(z+d+N)>-n$ we conclude that $\left\langle q_{z}(\xi), f(\xi)\right\rangle$ extends to a meromorphic function on the whole complex plane. Away from the poles, $q(z)$ is a homogeneous distribution of degree $z+d$ and in the distributional sense,

$$
q_{z}(-\xi)=(-1)^{d} q_{z}(\xi)
$$

Poles can only occur when $z+d$ lies in the set

$$
-n,-n-1,-n-2, \ldots,
$$

and the residue of $q_{z}$ at $z=-n-d-j$ is

$$
\sum_{|\alpha|=j} \frac{(-1)^{|\alpha|} \partial_{\xi}^{\alpha} \delta_{0}}{2 \alpha !} \int_{|\theta|=1} q(\theta) \theta^{\alpha} d \sigma(\theta),
$$

where $\delta_{0}$ is the Dirac delta function at 0 . In particular if $q$ has regular parity there is no pole at $z=0$ and $q_{0}$ is a regular parity homogeneous distribution of degree $d$ which equals $q$ away from the origin.

For the uniqueness, if $q^{\prime}$ and $q^{\prime \prime}$ are two homogeneous distributions of regular parity which equal $q$ away from zero, then $q^{\prime}-q^{\prime \prime}$ is supported at zero, hence equals a differential operator applied to the delta function at zero. Since $n$ is odd we conclude that $q^{\prime}-q^{\prime \prime}$ has singular parity, but since it is the difference of regular parity distributions it also has regular parity and hence equals zero.

In considering pseudodifferential operators, it will be convenient to allow homogeneous symbols as well as smooth symbols, so we will define classes of symbols $q(x, \xi)$ which are smooth functions for $\xi \neq 0$ and classical conormal distributions with respect to the set $\xi=0$.

Definition 2.1.1. Write $S^{d}(\Omega)$ for the space of smooth symbols on $\Omega$ of order $d$, that is, the space of functions $q \in C^{\infty}\left(\Omega \times \mathbb{R}^{n}\right)$ satisfying the estimates (2.1.1)

$$
\sup _{x \in K,|\xi| \geq 1}|\xi|^{-d+|\beta|}\left|\partial_{x}^{\alpha} \partial_{\xi}^{\beta} q(x, \xi)\right|<\infty \quad \text { for each } \alpha, \beta \text {, and compact } K \subset \Omega \text {. }
$$

Definition 2.1.2. Write $S D^{d}(\Omega)$ for the space of order $d$ distributional symbols on $\Omega \times \mathbb{R}^{n}$ which are conormal with respect to $\Omega \times 0$, that is, the space of distributions $q \in \mathcal{D}^{\prime}\left(\Omega \times \mathbb{R}^{n}\right)$ such that $q(x, \xi)$ is smooth away from $\xi=0$, satisfies (2.2.1), and there exists $N \in \mathbb{N}$ with

$$
\xi^{\gamma} \partial_{x}^{\alpha} \partial_{\xi}^{\beta} q(x, \xi) \in C\left(\Omega \times \mathbb{R}^{n}\right) \quad \text { for all } \alpha, \beta, \gamma \quad \text { with }|\gamma|-|\beta|>N \text {. }
$$

Write $S D^{d}(\Omega, \mathbb{C}(N))$ for the space of $\mathbb{C}(N)$-valued distributions on $\Omega \times \mathbb{R}^{n}$ whose matrix entries are in $S D^{d}(\Omega)$. 
LEMma 2.1.3. If $q \in S D^{d}(\Omega)$ where $d<-n$, then

$$
\hat{q}, \quad \check{q} \in C^{-n-d-1}\left(\Omega \times \mathbb{R}^{n}\right) .
$$

Now we introduce pseudodifferential operators. Let $\pi: E \rightarrow M$ be an $N$-dimensional complex vector bundle over the closed compact $n$-dimensional manifold $M$ where $n$ is odd. We will generally work in a coordinate chart $\Omega$ of $M$ over which $E$ is trivial, so $\pi^{-1}(\Omega)$ is identified with $U \times \mathbb{C}^{N}$, where $U$ is a subset of $\mathbb{R}^{n}$.

$Q: C^{\infty}(E) \rightarrow C^{\infty}(E)$ is a pseudodifferential operator of order $d$, if for each local coordinate chart $\Omega$ over which $E$ is trivial there exists a symbol $q \in S^{d}(\Omega, \mathbb{C}(N))$ such that whenever $f \in C^{\infty}(E)$ is supported $\Omega$, in coordinates

$$
Q f(x)=\frac{1}{(2 \pi)^{n}} \int_{\mathbb{R}^{n}} \int_{\mathbb{R}^{n}} q(x, \xi) e^{i(x-y) \cdot \xi} f(y) d y d \xi .
$$

Now formally

$$
\begin{aligned}
Q f(x) & =\int_{\mathbb{R}^{n}}\left(\left(\frac{1}{2 \pi}\right)^{n} \int_{\mathbb{R}^{n}} q(x, \xi) e^{i(x-y) \cdot \xi} d \xi\right) f(y) d y \\
& =\int_{\mathbb{R}^{n}} \check{q}(x, x-y) f(y) d y .
\end{aligned}
$$

Interpreted distributionally this shows that the Schwartz kernel $K(Q, x, y)$ equals the distribution $\check{q}(x, x-y)$.

2.2. Canonical splitting of operators. Suppose throughout this section that the dimension $n$ is odd. Let $\pi: E \rightarrow M$ be an $N$-dimensional complex vector bundle over the closed compact $n$-dimensional manifold $M$ and let $Q: C^{\infty}(E) \rightarrow C^{\infty}(E)$ be an odd class pseudodifferential operator of order $d$ with symbol expansion

$$
q(x, \xi) \sim q_{d}(x, \xi)+q_{d-1}(x, \xi)+\ldots, \quad \xi \rightarrow \infty,
$$

where $q_{j}(x, \xi)$ is a homogeneous distribution of degree $j$ in $\xi$ with regular parity. Then

$$
\check{q}(x, w)-\sum_{j \geq-n-M} \check{q}_{j}(x, w) \in C^{M}\left(\Omega \times \mathbb{R}^{n}, \mathbb{C}(N)\right) .
$$

It is convenient at this point to label functions of $w$ by their homogeneity by setting

$$
S_{j}(x, w)=\check{q}_{-n-j}(x, w) .
$$

It is well-known that given an arbitrary power series at a point, there is a smooth function $f$ whose Taylor series equals that power series. The function can be constructed by multiplying each term in the power series by a suitable 
cut-off function. The same construction gives a distribution $S(x, w)$ which is smooth for $w \neq 0$ and satisfies

$$
S(x, w) \sim \sum_{j=-n-\alpha}^{\infty} S_{j}(x, w),
$$

in the sense of (1.2.8). Then $R(x, w):=\check{q}(x, w)-S(x, w)$ is smooth. Summarizing, we have

$$
K(Q, x, y)=S(x, x-y)+R(x, x-y) .
$$

where $R(x, w)$ is smooth and $S(x, w)$ has singular parity in $w$ as $w \rightarrow 0$.

Now we form operators $Q_{\text {reg }}$ and $Q_{\text {sing }}$ with $Q=Q_{\text {reg }}+Q_{\text {sing }}$ by patching together the kernels $R(x, x-y)$ and $S(x, x-y)$. Choose a finite partition of unity $\phi_{j}$ for $M$ such that the supports of $\phi_{j}$ and $\phi_{\ell}$, are either disjoint or contained in a coordinate patch $\Omega$ over which $E$ is trivial. If the supports are disjoint then $\phi_{j} Q \phi_{\ell}$ is smoothing and we set

$$
Q_{\mathrm{reg}}^{j \ell}=\phi_{j} Q \phi_{\ell}, \quad Q_{\mathrm{sing}}^{j \ell}=0 .
$$

If not, the supports are contained in a coordinate patch $\Omega$ and we set

$$
\begin{aligned}
Q_{\mathrm{reg}}^{j \ell} f(x) & =\int_{\Omega} \phi_{j}(x) R(x, x-y) \phi_{\ell}(y) f(y) d y, \\
Q_{\mathrm{sing}}^{j \ell} f(x) & =\int_{\Omega} \phi_{j}(x) S(x, x-y) \phi_{\ell}(y) f(y) d y,
\end{aligned}
$$

where $R$ and $S$ are as defined above. Now we set

$$
Q_{\mathrm{reg}}=\sum_{j, \ell} Q_{\mathrm{reg}}^{j \ell}, \quad Q_{\mathrm{sing}}=\sum_{j, \ell} Q_{\mathrm{sing}}^{j \ell} .
$$

See Definition 1.2.3 for the definition of purely singular operators.

LEMma 2.2.1. (a) The class of purely singular operators contains the differential operators and is closed under composition with differential operators.

(b) The adjoint of a purely singular operator is purely singular.

(c) The operator $Q_{\text {sing }}$ constructed above is purely singular.

(d) If $Q$ is purely singular then $\operatorname{KERD}(Q, x)=0$ so that $\operatorname{TR} Q=0$.

Corollary 2.2.2. If $\partial$ is a differential operator then

$$
\begin{aligned}
\operatorname{TR} Q & =\operatorname{TR} Q_{\mathrm{reg}}+\operatorname{TR} Q_{\text {sing }}=\operatorname{TR} Q_{\mathrm{reg}}, \\
\operatorname{TR} \partial Q & =\operatorname{TR} \partial Q_{\mathrm{reg}}+\operatorname{TR} \partial Q_{\text {sing }}=\operatorname{TR} \partial Q_{\mathrm{reg}} .
\end{aligned}
$$

Remark. $Q_{\text {reg }}$ and $Q_{\text {sing }}$ are only well-defined modulo smoothing operators whose integral kernels vanish to infinite order at the diagonal. 
Proof of Lemma 2.2.1. (a) First we notice that the identity operator $I$ is purely singular, since $K(I, x, y)=\delta(x-y)$, and the delta function $\delta(w)$ is even in $w$ and has homogeneity $-n$. Next notice that if $S(x, w)$ is purely singular as $w \rightarrow 0$ and $\psi(x, w)$ is smooth, then $S(x, w) \psi(x, w)$ is purely singular as $w \rightarrow 0$. Indeed,

$$
\begin{aligned}
& S(x, w) \sim \sum_{j=d}^{\infty} S_{j}(x, w) \\
& \psi(x, w) \sim \sum_{j=0}^{\infty} \psi_{j}(x, w) \text { with } \psi_{j}(x, w)=\sum_{|\alpha|=j} \frac{\partial_{w}^{\alpha} \psi(x, 0)}{\alpha !} w^{\alpha},
\end{aligned}
$$

where $S_{j}(x, w)$ is homogeneous of degree $j$ in $w$ with singular parity. Clearly $S_{k}(x, w) \psi_{j}(x, w)$ is homogeneous with singular parity in $w$, and

$$
S(x, w) \psi(x, w) \sim \sum_{j=d}^{\infty} \sum_{k+\ell=j} S_{k}(x, w) \psi_{\ell}(x, w) .
$$

We need to show that if

$$
S(x, w) \sim \sum_{j=d}^{\infty} S_{j}(x, w)
$$

where $S_{j}(x, w)$ is homogeneous of order $j$ in $w$ with singular parity, then for a differential operator $P\left(x, D_{x}\right)=\sum_{|\beta| \leq N} a_{\beta}(x) \partial_{x}^{\beta}$,

$$
P\left(x, D_{x}\right) S_{j}(x, x-w) \sim \sum_{j=d-N}^{\infty} \tilde{S}_{j}(x, x-y)
$$

where $S_{j}(x, w)$ is homogeneous of order $j$ in $w$ with singular parity. We have already seen in (2.2.3) that $(2.2 .4)$ holds when $P\left(x, D_{x}\right)$ is multiplication by a smooth function. By induction it suffices to prove (2.2.4) for $P\left(x, D_{x}\right)$ $=\partial_{x_{k}}$, and this case follows easily by application of the chain rule to each term $S_{j}(x, x-y)$. We leave the details for the reader.

We omit the proof of (b), since it is not used in what follows.

To show (c), we want to see that $Q_{\text {sing }}^{j \ell}$ has singular parity. Now if the supports of $\phi_{j}$ and $\phi_{\ell}$ are not disjoint, then in some coordinate patch $\Omega$,

$$
K\left(Q_{\text {sing }}^{j \ell}, x, y\right)=\phi_{j}(x) S(x, x-y) \phi_{\ell}(y)=S^{j \ell}(x, x-y),
$$

where $S(x, w)$ has singular parity in $w$ as $w \rightarrow 0$. By part (a), $S^{j \ell}(x, w)$ also has singular parity in $w$ as $w \rightarrow 0$. We will now show that $S(x, w)$ still has singular parity under a change of coordinates. To simplify the presentation we fix $x$. It is a trivial modification to include the dependence on $x$. Suppose that 
$\Omega \subset \mathbb{R}^{n}$ is open and contains zero, and suppose that $S \in \mathcal{D}^{\prime}\left(\mathbb{R}^{n}\right)$ is compactly supported in $\Omega$, smooth away from 0 , and

$$
S(w) \sim \sum_{j \geq d} S_{j}(w), \quad w \rightarrow 0,
$$

where $S_{j}(w)$ is a homogeneous distribution of degree $j$ with singular parity. Let $F: U \rightarrow \Omega$ be a diffeomorphism with $F(0)=0$, and set $w=F(W)$. We wish to show that

$$
S(F(W)) \sim \sum_{j \geq d} \tilde{S}_{j}(W), \quad W \rightarrow 0
$$

where $\tilde{S}_{j}(W)$ is a homogeneous distribution of degree $j$ with singular parity in $W$. We start by showing that this is true away from $W=0$, which in fact proves the result in the case when $d>-n$. We will assume that there is only one term in the expansion of $S$, so that $S$ is homogeneous of degree $j$ for $w$ small. The general case follows by summing over $j$. Following a similar calculation in $[\mathrm{KV}]$, by Taylor's theorem, we have

$$
\begin{aligned}
F(W) & =F^{\prime}(0) W+\sum_{2 \leq|\alpha|<N} \frac{\partial^{\alpha} F(0)}{\alpha !} W^{\alpha}+O\left(|W|^{N}\right), \\
S\left(w_{0}+h\right) & =\sum_{|\beta|<N} \frac{\partial^{\beta} S\left(w_{0}\right)}{\beta !} h^{\beta}+O\left(|h|^{N}\left|w_{0}\right|^{j-N}\right) .
\end{aligned}
$$

Hence, setting $w_{0}=F^{\prime}(0) W$ and $h=F(W)-F^{\prime}(0) W$,

$$
S(F(W))=\sum_{|\beta|<N} \frac{\partial^{\beta} S\left(F^{\prime}(0)(W)\right)}{\beta !}\left(\sum_{2 \leq|\alpha|<N} \frac{\partial^{\alpha} F(0)}{\alpha !} W^{\alpha}\right)^{\beta}+O\left(|W|^{N+j-1}\right) .
$$

Moreover, if the equation is differentiated in $W$ any number of times, the error $O\left(|W|^{N+j-1}\right)$ is reduced in order by the number of $W$-derivatives. By grouping terms on the right-hand side of (2.2.6) which are homogeneous of degree $\ell$, we get (2.2.5).

In the case when $d \leq-n$, write

$$
S(w)=\left(1+\Delta_{w}\right)^{N} S_{0}(w)
$$

where $S_{0}$ has singular parity as $w \rightarrow 0$, is integrable and smooth away from zero, and $N$ is a positive integer. This is easily accomplished by using the Fourier transform. A change of variables gives

$$
S(F(W))=P\left(W, D_{W}\right) S_{0}(F(W))
$$


where

$$
P\left(W, D_{W}\right)=\left(1-\sum_{j, k, \ell} A_{k j}(W) \frac{\partial}{\partial W_{k}} A_{\ell j}(W) \frac{\partial}{\partial W_{\ell}}\right)^{N}, A(W)=\left(F^{\prime}(W)\right)^{-1} .
$$

We have already shown that $S_{0}(F(W))$ has singular parity in $W$ as $W \rightarrow 0$. Hence so does $S(F(W)$ ) by part (a). This completes the proof of (c). The same argument shows that the property of being a regular parity function or distribution is invariant under a change of coordinates.

Finally, we prove (d). Suppose $Q$ is a purely singular odd class operator of order $d$. Let $x \in M$, choose a coordinate chart $\Omega$ containing $x$ and let $q \in S^{d}(\Omega, \mathbb{C}(N))$ be the symbol of $Q$. Let $\phi$ be a smooth positive even function on $(-\infty, \infty)$ with

$$
\phi(r)=r, \quad r>1, \quad \phi(r)=1, \quad|r|<1 / 2 .
$$

Then for $x \in \Omega$, define an analytic family of operators $Q(z)$ so that on a neighborhood of $x, Q(z)$ has symbol

$$
q_{z}(x, \xi)=q(x, \xi) \phi(|\xi|)^{z} .
$$

Then

$$
\operatorname{KERD}(Q, x)=\left.\frac{1}{(2 \pi)^{n}} \int_{\mathbb{R}^{n}} q(x, \xi) \phi(|\xi|)^{z} d \xi\right|_{z=0} ^{\text {mer }} .
$$

We wish to show that this vanishes. Suppose $q$ has the expansion (2.2.1). We will compute

$$
\left.\frac{1}{(2 \pi)^{n}} \int_{\mathbb{R}^{n}} q_{j}(\xi)(\phi(|\xi|))^{z} d \xi\right|_{z=0} ^{\text {mer }} .
$$

If $j>-n$ and $\Re z<-j-n$ then

$$
\begin{aligned}
\int_{|\xi|>1} q_{j}(\xi)(\phi(|\xi|))^{z} d \xi & =\int_{1}^{\infty} r^{j+z+n-1} d r \int_{|\theta|=1} q_{j}(\theta) d s(\theta) \\
& =-\frac{1}{j+z+n} \int_{|\theta|=1} q_{j}(\theta) d s(\theta) .
\end{aligned}
$$

When we analytically continue to $z=0$ this equals

$$
-\frac{1}{j+n} \int_{|\theta|=1} q_{j}(x, \theta) d s(\theta) \text {. }
$$

On the other hand

$$
\left.\int_{|\xi|<1} q_{j}(x, \xi)(\phi(|\xi|))^{z} d \xi\right|_{z=0}=\int_{|\xi|<1} q_{j}(x, \xi) d \xi=\frac{1}{j+n} \int_{|\theta|=1} q_{j}(x, \theta) d s(\theta) .
$$


Hence (2.2.7) equals zero. If $j=-n$ then the distribution $q_{j}(x, \xi)(\phi(|\xi|))^{z}$ is odd in $\xi$ and hence integrates to zero when $\Re z<<0$. Hence again, (2.2.7) vanishes. Now set

$$
q^{(n)}(x, \xi)=q(x, \xi)-\sum_{j \geq-n} q_{j}(x, \xi) \in S^{-n-1}\left(\Omega, \mathbb{C}^{N \times N}\right) .
$$

Then $\int_{M} q^{(n)}(x, \xi) d \xi=0$. Indeed, $\check{q}(x, w)$ has singular parity in $w$ as $w \rightarrow 0$, hence so does $\check{q}^{(n)}(x, w)$, and

$$
\begin{aligned}
\frac{1}{(2 \pi)^{2} n} \int_{\mathbb{R}^{n}} q^{(n)}(x, \xi) e^{i \xi \cdot w} d \xi & =\check{q}^{(n)}(x, w) \\
& =\check{q}(x, w)-\sum_{j \geq-n} \check{q}_{j}(x, w) \in C\left(\Omega \times \mathbb{R}^{n}\right),
\end{aligned}
$$

which vanishes as $w \rightarrow 0$.

\subsection{Canonical trace for products.}

Proof of Theorem 1.2.6. Suppose that $P=\phi P \psi$ where $\phi$ and $\psi$ are functions on $M$ with disjoint supports. Then $P$ and $P A Q$ are smoothing and

$$
\begin{aligned}
T A(x)=\operatorname{KERD}(P A Q, x) & =K(P A Q, x, x) \\
& =\int_{M} K(P, x, y) A(y) K(Q, y, x) d y .
\end{aligned}
$$

Hence $T$ is a smoothing operator with integral kernel $K(P, x, y) \otimes K(Q, y, x)$. The same result holds if $Q=\phi Q \psi$. By taking a suitable partition of unity we just need to prove Theorem 1.2.6 in the case when there exists a coordinate chart $\Omega$ over which the vector bundles $E_{i}$ are trivial, and

there exists $\phi$ supported in $\Omega$ with $P=\phi P \phi, \quad Q=\phi Q \phi, \quad A=\phi A \phi$.

Now writing $P, A, Q$ as matrices we have

$$
(P A Q)_{i \ell}=\sum_{j, k} P_{i j} A_{j k} Q_{k \ell}
$$

If we prove the scalar case of the theorem we see that the general case follows immediately by considering the matrix entries.

We assume then that $P, Q: C^{\infty}(M) \rightarrow C^{\infty}(M)$ are odd-class pseudodifferential operators, $A \in C^{\infty}(M)$ and we restrict attention to a coordinate chart on which (2.3.2) holds. Write $Q^{t}$ for the transpose of $Q$ with respect to the standard density $d x$ on the coordinate chart. We consider separately the following cases: 
(1) $P$ and $Q$ are smoothing.

(2) $P$ is purely singular and $Q$ is smoothing.

(3) $Q^{t}$ is purely singular and $P$ is smoothing.

(4) $P$ and $Q^{t}$ are purely singular with $\operatorname{ord} P+\operatorname{ord} Q<-n$.

(5) $P$ and $Q^{t}$ are purely singular and in local coordinates, $K(P, x, x-w)$ and $K\left(Q^{t}, x, x-w\right)$ are homogeneous in $w$ (for $w$ small).

The general case follows since

$$
P=P_{\text {sing }}+P_{\text {reg }}, \quad Q^{t}=Q_{\text {sing }}^{t}+Q_{\text {reg }}^{t},
$$

and

$P_{\text {sing }}=P_{1}+\ldots+P_{j}+P^{(j)}, \quad Q_{\text {sing }}^{t}=Q_{1}+\ldots+Q_{j}+Q^{(j)}$

where $P^{(j)}, Q^{(j)}$ have low order and $K\left(P_{k}, x, x-w\right), K\left(Q_{k}, x, x-w\right)$ are homogeneous in $w$.

Case (1). If $P$ and $Q$ are both smoothing then (2.3.1) holds and $T$ is a smoothing operator.

Case (2). Similarly, when $P$ is purely singular and $Q$ is smoothing, $P A Q$ is smoothing and

$$
\begin{aligned}
T A(x) & =K(P A Q, x, x) \\
& =\langle K(P, x, \cdot), A(\cdot) K(Q, \cdot, x)\rangle=\langle K(P, x, \cdot) K(Q, \cdot, x), A(\cdot)\rangle .
\end{aligned}
$$

The product $K(P, x, x-w) K(Q, x-w, x)$ has singular parity in $w$ as $w \rightarrow 0$, and the lowest homogeneity occurring in $w$ is at least $-n-\operatorname{ord} P$. Hence $T$ is an odd class pseudodifferential operator with $\operatorname{ord} T \leq \operatorname{ord} P$.

Case (3). follows in the same way as Case (2), or indeed can be deduced from Case (2) because

$$
\begin{aligned}
T A(x) & =\operatorname{KERD}(P A Q, x) \\
& =K(P A Q, x, x)=K\left(Q^{t} A P^{t}, x, x\right)=\operatorname{KERD}\left(Q^{t} A P^{t}, x\right) .
\end{aligned}
$$

Here, $T$ is an odd class pseudodifferential operator with $\operatorname{ord} T \leq \operatorname{ord} Q$.

Case (4). When $P$ and $Q$ are both purely singular and $\operatorname{ord} P+\operatorname{ord} Q<-n$, the operator $P A Q$ is trace class and the function $K(P, x, y) K(Q, y, x)$ is integrable. A simple continuity argument shows that (2.3.1) is still valid. The kernel $K(P, x, y) K(Q, y, x)$ has an expansion in homogeneous terms in $y-x$ in local coordinates and so $T$ is a classical pseudodifferential operator. 
Case (5). Using local coordinates to work in $\mathbb{R}^{n}$ and reducing the support of $A$ if necessary, we assume that $K(P, x, x-w)$ and $K\left(Q^{t}, x, x-w\right)$ are homogeneous distributions in $w$ for $w \in \mathbb{R}^{n}$. Let $p(x, \xi)$ and $q^{t}(x, \xi)$ be the symbols of $P$ and $Q^{t}$ respectively, which are homogeneous in $\xi$. In a neighborhood of some point $x$, define the meromorphic families $P(z)$ and $Q(z)$ so that close to $x$ the symbols of $P(z)$ and $Q^{t}(z)$ are $p(z, x, \xi)$ and $q^{t}(z, x, \xi)$ respectively, where

$$
p(z, x, \xi)=p(x, \xi)|\xi|^{z / 2}, \quad \quad q^{t}(z, x, \xi)=q^{t}(x, \xi)|\xi|^{z / 2} .
$$

As shown in Section 2.1, this defines distributions which vary meromorphically with $z$ and have poles lying in the set $\mathbb{Z}+1 / 2$. We will show that

$$
\operatorname{KERD}(P A Q, x)=\left.K(P(z) A Q(z), x, x)\right|_{z=0} ^{\mathrm{mer}} .
$$

Indeed, let $\phi \in C^{\infty}(\mathbb{R})$ be positive and even with

$$
\phi(r)=r, \quad r>1, \quad \phi(r)=1, \quad|r|<1 / 2 .
$$

Define $P_{1}(z), Q_{1}^{t}(z)$ with symbols $p_{1}(z, x, \xi), q_{1}^{t}(z, x, \xi)$ by

$$
p_{1}(z, x, \xi)=p(x, \xi)(\phi(|\xi|))^{z / 2}, \quad q_{1}^{t}(z, x, \xi)=q^{t}(x, \xi)(\phi(|\xi|))^{z / 2} .
$$

Then $P_{1}(z)$ and $Q_{1}(z)$ vary analytically in $z$ and so

$$
\operatorname{KERD}(P A Q, x)=\left.K\left(P_{1}(z) A Q_{1}(z), x, x\right)\right|_{z=0} ^{\text {mer }} .
$$

However, $P(z)-P_{1}(z)$ and $Q(z)-Q_{1}(z)$ are smoothing operators depending meromorphically on $z$ with poles in $\mathbb{Z}+1 / 2$, and $P(0)-P_{1}(0)=0$, $Q(0)-Q_{1}(0)=0$. Hence

$$
\left.K\left(\left(P(z)-P_{1}(z)\right) A Q_{1}(z), x, x\right)\right|_{z=0} ^{\mathrm{mer}}=K\left(\left(P(0)-P_{1}(0)\right) A Q_{1}(0), x, x\right)=0,
$$

and similarly

$$
\left.K\left(P_{1}(z) A\left(Q(z)-Q_{1}(z)\right), x, x\right)\right|_{z=0} ^{\text {mer }}=0
$$

and

$$
\left.K\left(\left(P(z)-P_{1}(z)\right) A\left(Q(z)-Q_{1}(z)\right), x, x\right)\right|_{z=0} ^{\text {mer }}=0
$$

so that (2.3.3) follows. Now for $\Re z<<0$,

$$
\begin{aligned}
K(P(z) A Q(z), x, x) & =\int_{\mathbb{R}^{n}} K(P(z), x, y) A(y) K(Q(z), y, x) \\
& =\int_{\mathbb{R}^{n}} K(P(z), x, y) K\left(Q^{t}(z), x, y\right) A(y) .
\end{aligned}
$$

But $K(P(z), x, x-w) K\left(Q^{t}(z), x, x-w\right)$ is homogeneous in $w$ of degree $-2 n-\operatorname{ord} P-\operatorname{ord} Q-z$ and is an even function of $w$. From the proof of Lemma 1.2.5 given in Section 2.1, we see that as a distribution, this product 
meromorphically continues and gives a regular parity distribution at $z=0$, which is the kernel of a pseudodifferential operator of order $n+\operatorname{ord} P+\operatorname{ord} Q$. This completes the proof of (5). The symbol of $T$ in this case is $\left(\check{p} \breve{q}^{t}\right)^{\wedge}(x, \xi)$, but $q^{t}(x, \xi)=q(x,-\xi)$ where $q$ is the principal symbol of $Q$.

\section{Variation formulas}

Throughout this section, $g_{0}$ is a metric on the $n$-dimensional manifold $M$ where $n$ is odd. $V_{0}$ is the total volume of $M$ in the metric $g_{0}$ and $d \mu_{0}$ is the canonical volume element $g_{0}$, scaled to have total volume one. $\Pi_{0}$ is the $d \mu_{0}$-orthogonal projection of $L^{2}(M)$ onto the constants, and $\Delta_{0}=\Delta_{g_{0}}$. The components of tensor fields are given with respect to a local orthonormal frame for $g_{0}$, and $D_{i}$ denotes $g_{0}$-covariant differentiation in the direction of the $i^{\text {th }}$ frame vector. Consider a deformation $g(t)$ of $g_{0}$ and write

$$
\begin{aligned}
& g(t)=e^{2 f(t)} B(t), \quad \text { where } \quad \operatorname{det} B_{i j}=1, \quad f=\frac{1}{2 n} \log \operatorname{det} g_{i j}, \\
& A_{i j}=\dot{B}_{i j}(0), \quad \phi=\dot{f}(0) .
\end{aligned}
$$

Write $V$ for the total volume of $M$ in the metric $g$ and $\Pi$ for the orthogonal projection onto the constants in the metric $g$.

3.1. First variation of $\operatorname{det}^{\prime} \Delta$. Our goal is to compute the first variation of the homogenized log determinant functional

$$
F=\log \operatorname{det}^{\prime} \Delta-\frac{2}{n} \log V,
$$

and use this to characterize the critical metrics.

Write $G(x, y)$ for the Green function for $\Delta_{0}$ and $\mathcal{G}(x, y)$ for the regular part of the Green function, so that $\mathcal{G}(x, y) d \mu_{0}$ is the Schwartz kernel for $\left(\Delta_{0}^{-1}\right)_{\text {reg. }}$. With the notation of (3.0.1), set

$$
E=e^{(n-2) f} B^{-1} .
$$

Then

$$
\begin{array}{ll}
\Pi=\left(\Pi_{0} e^{2 f}\right)^{-1} \Pi_{0} e^{2 f}, & \dot{\Pi}(0)=2 \Pi_{0}\left(\phi-\left(\Pi_{0} \phi\right)\right), \\
\Delta=-e^{-n f} D_{i} E_{i j} D_{j}, & \dot{\Delta}(0)=-n \phi \Delta_{0}-D_{i} \dot{E}_{i j}(0) D_{j} .
\end{array}
$$

Proof of Theorem 1.3.6.

$$
\begin{aligned}
\frac{d}{d t} \log \operatorname{det} \Delta & =\operatorname{TR}(\dot{\Delta}+\dot{\Pi})(\Delta+\Pi)^{-1}=\operatorname{TR} \dot{\Delta}\left(\Delta^{-1}+\Pi\right)+\operatorname{TR} \dot{\Pi}\left(\Delta^{-1}+\Pi\right) \\
& =\operatorname{TR} \dot{\Delta} \Delta^{-1}+\operatorname{TR} \dot{\Pi} \Pi=\operatorname{TR} \dot{\Delta} \Delta^{-1}
\end{aligned}
$$


The first equality follows from [Ok1], and the last from the fact that TR $\frac{1}{2} \operatorname{trace}\left(\Pi^{2}\right)^{\cdot}=\frac{1}{2}(\operatorname{trace} \Pi)^{\cdot}=0$. Now $V=\left(\Pi_{0} e^{n f}\right) V_{0}$, so that

$$
\begin{aligned}
\left.\frac{d F}{d t}\right|_{t=0} & =\operatorname{TR} \dot{\Delta}_{0} \Delta_{0}^{-1}-2\left(\Pi_{0} \phi\right) \\
& =\operatorname{TR}\left(-n \phi \Delta_{0}-D_{i} \dot{E}_{i j}(0) D_{j}\right) \Delta_{0}^{-1}-2\left(\Pi_{0} \phi\right) \\
& =(n-2) \operatorname{TR} \phi \Pi_{0}-\operatorname{TR} D_{i} \dot{E}_{i j}(0) D_{j} \Delta_{0}^{-1} \\
& =(n-2) \int_{M} \phi d \mu_{0}+\left.\int_{M} \dot{E}_{i j}(0)(x) D_{y, i} D_{x, j} \mathcal{G}(x, y)\right|_{y=x} d \mu_{0} \\
& =\int_{M} \dot{E}_{i j}(0)(x)\left(\left.D_{y, i} D_{x, j} \mathcal{G}(x, y)\right|_{y=x}+\frac{1}{n} \delta_{i j}\right) d \mu_{0} .
\end{aligned}
$$

Here, we have integrated by parts using the fact that for a tensor field $H(x, y)$,

$$
D_{i}\left(\left.H(x, y)\right|_{y=x}\right)=\left.D_{x, i} H(x, y)\right|_{y=x}+\left.D_{y, i} H(x, y)\right|_{y=x} .
$$

We have also used the fact that

$$
\dot{E}_{i j}(0)=(n-2) \phi \delta_{i j}-A_{i j}(0),
$$

so that

$$
(n-2) \phi=\frac{\dot{E}_{k k}}{n}
$$

Proof of Corollary 1.3.7. From Theorem 1.3.6, we see that the metric $g_{0}$ is critical for $F$ if and only if (1.3.2) holds; that is,

$$
\left.D_{y, i} D_{x, j} \mathcal{G}(x, y)\right)\left.\right|_{y=x}+\frac{\delta_{i j}}{n}=0 .
$$

To see that this implies (1.3.3), we first note that

$$
\begin{aligned}
\left.\left(\Delta_{0, x} \mathcal{G}(x, y)\right)\right|_{y=x} & =-1 . \\
\left.D_{y, i} D_{x, j} \mathcal{G}(x, y)\right)\left.\right|_{y=x} & =\frac{1}{2} D_{i} D_{j}(\mathcal{G}(x, x))-\left.\left(D_{x, i} D_{x, j} \mathcal{G}(x, y)\right)\right|_{y=x} . \\
\left.D_{y, i} D_{x, i} \mathcal{G}(x, y)\right)\left.\right|_{y=x} & =-\frac{1}{2} \Delta_{0}(\mathcal{G}(x, x))-1 .
\end{aligned}
$$

Indeed (3.1.4) follows since

$$
\Delta_{0, x} G(x, y)=-1, \quad y \neq x .
$$

To see (3.1.5) note that

$$
\left.D_{x} \mathcal{G}(x, y)\right|_{y=x}=\left.D_{y} \mathcal{G}(x, y)\right|_{y=x},
$$


which follows since $\mathcal{G}(x, y)$ with $\mathcal{G}(y, x)=\mathcal{G}(x, y)$. By the chain rule,

$$
D(\mathcal{G}(x, x))=\left.D_{x} \mathcal{G}(x, y)\right|_{y=x}+\left.D_{y} \mathcal{G}(x, y)\right|_{y=x} .
$$

Putting these two facts together,

$$
\left.D_{x} \mathcal{G}(x, y)\right|_{y=x}=\left.D_{y} \mathcal{G}(x, y)\right|_{y=x}=\frac{1}{2} D(\mathcal{G}(x, x)) .
$$

Applying the chain rule to (3.1.7) gives (3.1.5), and setting $i=j$ in (3.1.5) gives (3.1.6). The condition (3.1.3) now becomes

$$
\frac{1}{2} D_{i} D_{j}(\mathcal{G}(x, x))-\left.\left(D_{x, i} D_{x, j} \mathcal{G}(x, y)\right)\right|_{y=x}+\frac{\delta_{i j}}{n}=0 .
$$

Setting $i=j$ and using (3.1.4) we get

$$
\frac{1}{2} \Delta_{0}(\mathcal{G}(x, x))=0 \text {. }
$$

Now, $\mathcal{G}(x, x)$ is constant, and (3.1.8) becomes

$$
\left.-D_{x, i} D_{x, j} \mathcal{G}(x, y)\right)\left.\right|_{y=x}+\frac{\delta_{i j}}{n}=0
$$

which is (1.3.3)(b). Clearly (1.3.3) implies (1.3.4). Now assume that (1.3.4) holds; that is, $\mathcal{G}(x, x)$ is constant and

$$
\left.D_{x, i} D_{x, j} \mathcal{G}(x, y)\right)\left.\right|_{y=x}=c \delta_{i j} .
$$

Setting $i=j$ and summing we get $c=1 / n$ which implies (3.1.8) which implies (3.1.3).

3.2. First variation of det $L$. In this section we calculate the first variation of $\log \operatorname{det} L$ and hence characterize the critical metrics for $\operatorname{det} L$.

Lemma 3.2.1. Let $g(t)$ be a deformation of the metric $g_{0}$ and set $h=\dot{g}(0)$. Then

$$
\left.\frac{d S}{d t}\right|_{t=0}=h_{i j, i j}-h_{i i, j j}-R_{i j} h_{i j}
$$

where $R_{i j}$ is the Ricci tensor for $g_{0}$.

A proof of this fact can be found in [LP, §8], or in Section 3.4 of this paper. Now if $g(t)$ is a conformal variation of $g_{0}$, and $g(t)=e^{2 f(t)} g_{0}$, then

$$
L=e^{-\left(\frac{n}{2}+1\right) f} L_{0} e^{\left(\frac{n}{2}-1\right) f} .
$$

It was shown in $[\mathrm{PR}]$ that in odd dimensions,

$$
\operatorname{det} L=\operatorname{det} L_{0} \text {. }
$$


Indeed, this can be easily deduced using the canonical trace:

$$
\begin{aligned}
\frac{d}{d t} \log \operatorname{det} L & =\operatorname{TR} \dot{L} L^{-1} \\
& =\operatorname{TR}\left(\left(-\frac{n}{2}-1\right) \dot{f} L+\left(\frac{n}{2}-1\right) L \dot{f}\right) L^{-1}=-2 \operatorname{TR} \dot{f}=0
\end{aligned}
$$

Because of this, it is sufficient to consider variations $g(t)$ which preserve the volume element, so that $\dot{g}(0)=A$ where $A_{i i}=0$. We have

$$
\left.\frac{d}{d t}\right|_{t=0}(\log \operatorname{det} L)=\operatorname{TR} \dot{L}(0) L_{0}^{-1} .
$$

Lemma 3.2.1 gives

$$
\dot{S}(0)=A_{i j, i j}-R_{i j} A_{i j}, \quad \dot{\Delta}(0)=D_{i} A_{i j} D_{j}=A_{i j} D_{i} D_{j}+A_{i j, i} D_{j} .
$$

Proof of Theorem 1.3.8.

$$
\begin{aligned}
\left.\frac{d}{d t}\right|_{t=0} \log \operatorname{det} L & =\operatorname{TR} \dot{L}(0) L_{0}^{-1} \\
& =\operatorname{TR}\left(A_{i j} D_{i} D_{j}+A_{i j, i} D_{j}+\alpha\left(A_{i j, i j}-R_{i j} A_{i j}\right)\right) L_{0}^{-1} \\
& =\int_{M}\left(\left.A_{i j} D_{x, i} D_{x, j} \mathcal{G}(x, y)\right|_{y=x}+\left.A_{i j, i} D_{x, j} \mathcal{G}(x, y)\right|_{y=x}\right. \\
& \left.+\alpha\left(A_{i j, i j}-R_{i j} A_{i j}\right) \mathcal{G}(x, x)\right) d \mu_{0} \\
& =\int_{M}\left(\left.A_{i j}\left(D_{x, i} D_{x, j}-\alpha R_{i j}\right) \mathcal{G}(x, y)\right|_{y=x}\right. \\
& \left.+\left(\alpha-\frac{1}{2}\right) A_{i j} D_{i} D_{j}(\mathcal{G}(x, x))\right) d \mu_{0} \\
& =\int_{M} A_{i j}(x) Q_{i j}(x) d \mu_{0}(x)
\end{aligned}
$$

where

$$
Q_{i j}(x)=\left.\left(D_{x, i} D_{x, j}-\alpha R_{i j}\right) \mathcal{G}(x, y)\right|_{y=x}-\frac{n}{4(n-1)} D_{i} D_{j}(\mathcal{G}(x, x)) .
$$

So $g_{0}$ is critical for $\operatorname{det} L$ if

$$
Q_{i j}(x)=C(x) \delta_{i j}
$$


for some function $C(x)$ which can be computed by contracting over $i$ and $j$ and using the fact that $\left.L_{x} G(x, y)\right|_{y=x}=0$. We get

$$
C(x)=\frac{1}{4(n-1)} \Delta_{0}(\mathcal{G}(x, x)),
$$

so $g_{0}$ is critical for $\operatorname{det} L$ if and only if

$$
\left.\left(D_{x, i} D_{x, j}-\alpha R_{i j}\right) \mathcal{G}(x, y)\right|_{y=x}=\frac{1}{4(n-1)}\left(n D_{i} D_{j}+\delta_{i j} \Delta_{0}\right)(\mathcal{G}(x, x)) .
$$

For later use we note that if $g_{0}$ is critical for $\operatorname{det} L$ then for any $Z \in T_{g_{0}} \mathcal{M}$,

$$
\operatorname{TR}\left(D_{i} Z_{i j} D_{j}-\alpha Z_{i j} R_{i j}+\alpha Z_{i j, i j}\right) L_{0}^{-1}=-\frac{1}{4(n-1)} \operatorname{TR} Z_{i i, j j} L_{0}^{-1} .
$$

\subsection{Second variation of $\operatorname{det}^{\prime} \Delta$.}

Proof of Theorem 1.3.10. We want to calculate the Hessian of $F$ at a critical metric $g_{0}$, and we do so in three steps. First we calculate the conformal case Hess $F\left(2 \phi g_{0}, 2 \phi g_{0}\right)$. Then we calculate the cross-term Hess $F\left(2 \phi g_{0}, A\right)$ when $A_{i i}=0$, and finally we calculate the volume element preserving the case $\operatorname{Hess}(A, A)$.

Recall that

$$
\bar{\phi}=\phi-\int_{M} \phi d \mu_{0}, \quad \bar{\Delta}_{0}^{-1}=\Delta_{0}^{-1}-Z(1) \Pi_{0} .
$$

We will start by showing that

$$
\begin{aligned}
\operatorname{Hess} F\left(2 \phi g_{0}, 2 \phi g_{0}\right)=\frac{(n+2)(n-2)}{2} & \int_{M} \bar{\phi}^{2} d \mu_{0} \\
& -\frac{(n-2)^{2}}{4} \mathrm{TR}\left(\left(\Delta_{0} \phi\right) \bar{\Delta}_{0}^{-1}\right)^{2} .
\end{aligned}
$$

Set

$$
g(t)=e^{2 t \phi} g_{0}
$$

Now

$$
\operatorname{Hess} F\left(2 \phi g_{0}, 2 \phi g_{0}\right)=\left.\frac{d^{2} F}{d t^{2}}\right|_{t=0}
$$

and as in (3.1.1),

$$
\left.\frac{d F}{d t}\right|_{t=0}=\operatorname{TR} \dot{\Delta} \Delta^{-1}-2(\Pi \phi) .
$$


Now

$$
\begin{aligned}
\Delta & =-e^{-n t \phi} D_{i} e^{(n-2) t \phi} D_{i}, \\
\dot{\Delta}(0) & =n \phi D_{i} D_{i}-(n-2) D_{i} \phi D_{i} \\
& =-\frac{n+2}{2} \phi \Delta_{0}+\frac{n-2}{2} \Delta_{0} \phi-\frac{n-2}{2}\left(\Delta_{0} \phi\right),
\end{aligned}
$$

where we temporarily use the slightly unwieldy convention that $\left(\Delta_{0} \phi\right)$ is the operator $\Delta_{0}$ applied to the function $\phi$, while $\Delta_{0} \phi$ is the operator $\Delta_{0}$ composed with multiplication by $\phi$. Since there is nothing special about $t=0$, we get

$$
\dot{\Delta}=-\frac{n+2}{2} \phi \Delta+\frac{n-2}{2} \Delta \phi-\frac{n-2}{2}(\Delta \phi) .
$$

Then

$$
\begin{aligned}
\operatorname{TR} \dot{\Delta} \Delta^{-1} & =\operatorname{TR}\left(-\frac{n+2}{2} \phi \Delta+\frac{n-2}{2} \Delta \phi-\frac{n-2}{2}(\Delta \phi)\right) \Delta^{-1} \\
& =-2 \operatorname{TR} \phi(I-\Pi)-\frac{n-2}{2} \operatorname{TR}(\Delta \phi) \Delta^{-1} \\
& =2 \operatorname{TR} \phi \Pi-\frac{n-2}{2} \operatorname{TR}(\Delta \phi) \Delta^{-1} .
\end{aligned}
$$

Thus

$$
\frac{d F}{d t}=-\frac{n-2}{2} \operatorname{TR}(\Delta \phi) \Delta^{-1}
$$

A similar formula was obtained by Richardson in [Ri] using more elaborate calculations involving the heat kernel instead of the canonical trace. Now

$$
\operatorname{TR}(\Delta \phi) \Pi=\int_{M} \Delta \phi d \mu=0 .
$$

Hence for any choice of $\beta$,

$$
\frac{d F}{d t}=-\frac{n-2}{2} \operatorname{TR}(\Delta \phi)(\Delta+\beta \Pi)^{-1}
$$

Let $Z(s)$ be the zeta function for $\Delta_{0}$ set

$$
\beta=-\frac{1}{Z(1)}, \quad \bar{\Delta}=\Delta+\beta \Pi, \quad \bar{\Delta}_{0}=\Delta_{0}+\beta \Pi_{0} .
$$

(We assume that $Z(s) \neq 0$. In the case $Z(s)=0$, the following argument can be modified by taking $\beta$ to be a constant and letting $|\beta| \rightarrow \infty$ at the end.) Since $g_{0}$ is critical for $F$ we have $\mathcal{G}(x, x)=Z(1)$ for all $x$, so that

$$
\bar{\Delta}_{0}^{-1}=\Delta_{0}^{-1}-Z(1) \Pi_{0}, \quad K\left(\left(\bar{\Delta}^{-1}\right)_{\mathrm{reg}}, x, x\right)=\mathcal{G}(x, x)-Z(1)=0,
$$

and $\operatorname{TR} \psi \bar{\Delta}_{0}^{-1}=0$ for any function $\psi$. Now

$$
\Pi_{0} \bar{\Delta}_{0}^{-1}=\bar{\Delta}_{0}^{-1} \Pi_{0}=-Z(1) \Pi_{0}, \quad \Delta_{0} \bar{\Delta}_{0}^{-1}=\bar{\Delta}_{0}^{-1} \Delta_{0}=I-\Pi_{0} .
$$


Hence

$$
\begin{aligned}
\left.\frac{d^{2} F}{d t^{2}}\right|_{t=0} & =-\frac{n-2}{2}\left(\operatorname{TR}(\dot{\Delta}(0) \phi) \bar{\Delta}_{0}^{-1}+\operatorname{TR}\left(\Delta_{0} \phi\right)\left((\Delta+\beta \Pi)^{-1}\right)^{\cdot}(0)\right) \\
& =-\frac{n-2}{2} \operatorname{TR}\left(\Delta_{0} \phi\right)\left((\Delta+\beta \Pi)^{-1}\right)^{\cdot}(0) \\
& =\frac{n-2}{2} \operatorname{TR}\left(\Delta_{0} \phi\right) \bar{\Delta}_{0}^{-1}\left(\dot{\Delta}_{0}+\beta \dot{\Pi}_{0}\right) \bar{\Delta}_{0}^{-1} \\
& =\frac{n-2}{2}\left(\beta \operatorname{TR}\left(\Delta_{0} \phi\right) \bar{\Delta}_{0}^{-1} \dot{\Pi}_{0} \bar{\Delta}_{0}^{-1}+\operatorname{TR}\left(\Delta_{0} \phi\right) \bar{\Delta}_{0}^{-1} \dot{\Delta}_{0} \bar{\Delta}_{0}^{-1}\right) .
\end{aligned}
$$

But

$$
\begin{aligned}
\beta \operatorname{TR}\left(\Delta_{0} \phi\right) \bar{\Delta}_{0}^{-1} \dot{\Pi}_{0} \bar{\Delta}_{0}^{-1} & =\beta \beta^{-1} \operatorname{TR}\left(\Delta_{0} \phi\right) \Pi_{0} \dot{\Pi}_{0} \bar{\Delta}_{0}^{-1} \\
& =n \operatorname{TR}\left(\Delta_{0} \phi\right) \Pi_{0} \bar{\phi} \bar{\Delta}_{0}^{-1}=n \int \bar{\phi}^{2} d \mu_{0}
\end{aligned}
$$

while

$$
\begin{aligned}
& \operatorname{TR}\left(\Delta_{0} \phi\right) \bar{\Delta}_{0}^{-1} \dot{\Delta}_{0} \bar{\Delta}_{0}^{-1} \\
&=\operatorname{TR}\left(\Delta_{0} \phi\right) \bar{\Delta}_{0}^{-1}\left(-\frac{n+2}{2} \phi \Delta_{0}+\frac{n-2}{2} \Delta_{0} \phi-\frac{n-2}{2}\left(\Delta_{0} \phi\right)\right) \bar{\Delta}_{0}^{-1} \\
&=-\frac{n+2}{2} \operatorname{TR}\left(\Delta_{0} \phi\right) \bar{\Delta}_{0}^{-1} \phi\left(I-\Pi_{0}\right)+\frac{n-2}{2} \operatorname{TR}\left(\Delta_{0} \phi\right)\left(I-\Pi_{0}\right) \phi \bar{\Delta}_{0}^{-1} \\
&-\frac{n-2}{2} \operatorname{TR}\left(\Delta_{0} \phi\right) \bar{\Delta}_{0}^{-1}\left(\Delta_{0} \phi\right) \bar{\Delta}_{0}^{-1} \\
&=-2 \operatorname{TR}\left(\Delta_{0} \phi\right)\left(I-\Pi_{0}\right) \phi \bar{\Delta}_{0}^{-1}-\frac{n-2}{2} \operatorname{TR}\left(\Delta_{0} \phi\right) \bar{\Delta}_{0}^{-1}\left(\Delta_{0} \phi\right) \bar{\Delta}_{0}^{-1} \\
&= 2 \operatorname{TR}\left(\Delta_{0} \phi\right) \Pi_{0} \phi \bar{\Delta}_{0}^{-1}-\frac{n-2}{2} \operatorname{TR}\left(\Delta_{0} \phi\right) \bar{\Delta}_{0}^{-1}\left(\Delta_{0} \phi\right) \bar{\Delta}_{0}^{-1} \\
&= 2 \operatorname{TR} \int_{M} \bar{\phi}^{2} d \mu_{0}-\frac{n-2}{2} \operatorname{TR}\left(\Delta_{0} \phi\right) \bar{\Delta}_{0}^{-1}\left(\Delta_{0} \phi\right) \bar{\Delta}_{0}^{-1} .
\end{aligned}
$$

Substitution of this and (3.3.6) into (3.3.5) gives (3.3.1).

Now we show that if $A_{i i}=0$ then

$$
\text { Hess } F\left(2 \phi g_{0}, A\right)=-\frac{n-2}{2} \operatorname{TR} \phi_{k k} \bar{\Delta}_{0}^{-1} D_{i} A_{i j} D_{j} \bar{\Delta}_{0}^{-1} .
$$

Take a deformation

$$
g(s, t)=e^{2 t \phi} B(s), \quad \operatorname{det}\left(B_{i j}\right)=1, \quad B_{i j}^{\prime}(0)=A_{i j},
$$

where the prime denotes differentiation with respect to $s$. With the notation of (3.3.4),

$$
\frac{\partial F}{\partial t}=-\frac{n-2}{2} \operatorname{TR}(\Delta \phi) \bar{\Delta}^{-1}
$$


Then

$$
\begin{aligned}
\left.\frac{\partial^{2} F}{\partial s \partial t}\right|_{s=t=0}= & -\frac{n-2}{2} \operatorname{TR}\left(\Delta^{\prime}(0,0) \phi\right) \bar{\Delta}_{0}^{-1} \\
& \quad-\frac{n-2}{2} \operatorname{TR}\left(\Delta_{0} \phi\right)\left((\Delta+\beta \Pi)^{-1}\right)^{\prime}(0,0) \\
= & -\frac{n-2}{2} \operatorname{TR} \phi_{k k} \bar{\Delta}_{0}^{-1} \Delta^{\prime}(0) \bar{\Delta}_{0}^{-1} \\
= & -\frac{n-2}{2} \operatorname{TR} \phi_{k k} \bar{\Delta}_{0}^{-1} D_{i} A_{i j} D_{j} \bar{\Delta}_{0}^{-1}
\end{aligned}
$$

Finally, we show that

$$
\operatorname{Hess} F(A, A)=-\frac{1}{n} \int_{M} A_{i j}^{2} d \mu_{0}-\operatorname{TR} D_{i} A_{i j} D_{j} \bar{\Delta}^{-1} D_{k} A_{k \ell} D_{\ell} \bar{\Delta}^{-1} .
$$

Taking a deformation $g(t)$ of $g_{0}$ with $\operatorname{det}\left(g_{i j}(t)\right)=1$ for all $t$ and $\dot{g}(0)=A$, we also set $Z_{i j}=\partial_{t}^{2}\left(g^{-1}\right)_{i j}(0)$ and note that since $\operatorname{det}\left(g_{i j}\right)=1$,

$$
Z_{i i}=A_{i j} A_{i j}
$$

Now with the notation of (3.3.4) we have

$$
\frac{d F}{d s}=\operatorname{TR} \Delta^{\prime} \bar{\Delta}^{-1}
$$

so that

$$
\left.\frac{d^{2} F}{d s^{2}}\right|_{s=0}=\operatorname{TR} \Delta^{\prime \prime}(0) \bar{\Delta}_{0}^{-1}-\operatorname{TR} \Delta^{\prime}(0) \bar{\Delta}_{0}^{-1} \Delta^{\prime}(0) \bar{\Delta}_{0}^{-1}
$$

Now

$$
\Delta^{\prime}(0)=D_{i} A_{i j} D_{j}, \quad \Delta^{\prime \prime}(0)=-D_{i} Z_{i j} D_{j},
$$

so that the second term on the right of (3.3.9) equals the second term on the right in (3.3.8). Now since $g_{0}$ is critical,

$$
\begin{aligned}
\operatorname{TR} \bar{\Delta}^{\prime \prime}(0) \Delta_{0}^{-1} & =-\operatorname{TR} D_{i} Z_{i j} D_{j} \bar{\Delta}_{0}^{-1}=\left.\int_{M} Z_{i j} D_{y, i} D_{x, j} \mathcal{G}(x, y)\right|_{y=x} d \mu_{0} \\
& =-\frac{1}{n} \int_{M} Z_{i j} \delta_{i j} d \mu_{0}=-\frac{1}{n} \int_{M} A_{i j} A_{i j} d \mu_{0}
\end{aligned}
$$

3.4. Second variation of $\operatorname{det} L$. In this section we prove Theorem 1.3.11. First we remark that this theorem enables us to compute Hess $\log \operatorname{det} L$ on the space $T_{g_{0}} \mathcal{M}$. Indeed,

$$
T_{g_{0}} \mathcal{M}=\left(\operatorname{conf} g_{0}+\operatorname{diff} g_{0}\right)^{\perp} \oplus\left(\operatorname{conf} g_{0}+\operatorname{diff} g_{0}\right) .
$$


Furthermore, $A \in\left(\operatorname{conf} g_{0}+\operatorname{diff} g_{0}\right)^{\perp}$ if and only if $A_{i i}=0, A_{i j, j}=0$, and Theorem 1.3.11 gives Hess $\log \operatorname{det} L$ under these conditions. We claim that if $X \in \operatorname{diff}\left(g_{0}\right)+\operatorname{conf}\left(g_{0}\right)$ and $Y \in T_{g_{0}} \mathcal{M}$, then Hess $\log \operatorname{det} L(X, Y)=0$. Indeed,

$$
\operatorname{Hess}(X, Y)=\left.\partial_{s} \partial_{t} F(g(s, t))\right|_{s=t=0}
$$

where $g(s, t)$ is a 2-parameter deformation of $g_{0}$ with $\partial_{s} g(0,0)=X$ and $\partial_{t} g(0,0)=Y$. Write $X=2 \phi \delta_{i j}+X_{1}$ where $X_{1} \in \operatorname{diff}\left(g_{0}\right)$. It is not hard to construct a deformation $h(s, t)$ of $g_{0}$ with $h(s, 0) \in \operatorname{Diff}\left(g_{0}\right)$ for all $s, \partial_{s} h(0,0)=X_{1}$ and $\partial_{t} h(0,0)=Y$. Set $g(s, t)=e^{2 s \phi} h(s, t)$. Then $\partial_{s} g(0,0)=X$ and $\partial_{t} g(0,0)=$ $Y$. But then $\log \operatorname{det} L(g(s, t)=\log \operatorname{det} L(h(s, t))$ for all $s$ and $t$, and so we can replace $g(s, t)$ by $h(s, t)$ on the right-hand side of (3.4.1), which then vanishes because $h(s, 0)$ is critical for $\log \operatorname{det} L$ so that $\left.\partial_{t} \log \operatorname{det} L(h(s, t))\right|_{t=0}=0$.

Proof of Theorem 1.3.11. We take a deformation of $g_{0}$ with $\dot{g}_{i j}(0)=A_{i j}$ where $A_{i i}=0$ and $A_{i j, j}=0$, and set $\left(g_{i j}^{-1}\right)^{\cdot}(0)=Z_{i j}$. By Lemma 3.2.1,

Hess log $\operatorname{det} L(A, A)$

$$
\begin{aligned}
& =\left.\frac{d^{2}}{d t^{2}}\right|_{t=0} \log \operatorname{det} L_{g(t)}=\operatorname{TR} \ddot{L}(0) L_{0}^{-1}-\operatorname{TR} L_{0}^{-1} \dot{L}(0) L_{0}^{-1} \dot{L}(0) \\
& =-\operatorname{TR} D_{i} Z_{i j} D_{j} L_{0}^{-1}+\alpha \operatorname{TR} \ddot{S}(0) L_{0}^{-1} \\
& \quad \quad-\operatorname{TR}\left(D_{i} A_{i j} D_{j}-\alpha R_{i j} A_{i j}\right) L_{0}^{-1}\left(D_{k} A_{k \ell} D_{\ell}-\alpha R_{k \ell} A_{k \ell}\right) L_{0}^{-1} .
\end{aligned}
$$

The last term in this final expression is the same as the last term in (1.3.11). To equate the other terms, we need the following.

Lemma 3.4.1. If $A_{i i}=0$ and $A_{i j, j}=0$, then

$$
\frac{d^{2} S}{d t^{2}}=Z_{i j} R_{i j}-Z_{i j, i j}+A_{i j, k} A_{i k, j}-\frac{1}{2} A_{i j, k} A_{i j, k} .
$$

Using this Lemma, equation (3.2.3), and the fact that $Z_{i i}=A_{i j} A_{i j}$, we see that

$$
\begin{aligned}
& -\operatorname{TR} D_{i} Z_{i j} D_{j} L_{0}^{-1}+\alpha \operatorname{TR} \ddot{S}(0) L_{0}^{-1} \\
& \quad=\operatorname{TR}\left(-D_{i} Z_{i j} D_{j}+\alpha Z_{i j} R_{i j}-\alpha Z_{i j, i j}+\alpha A_{i j, k} A_{i k, j}-\frac{\alpha}{2} A_{i j, k} A_{i j, k}\right) L_{0}^{-1} \\
& \quad=\operatorname{TR}\left(\frac{1}{4(n-1)}\left(A_{i j} A_{i j}\right)_{k k}+\alpha A_{i j, k} A_{i k, j}-\frac{\alpha}{2} A_{i j, k} A_{i j, k}\right) L_{0}^{-1} .
\end{aligned}
$$

Proof of Lemma 3.4.1. We work in coordinates. The metric $g(t)$ is a deformation of $g_{0}$. We denote the covariant derivative in the metric $g(t)$ by $\nabla=\nabla(t)$, so that $\nabla(0)=D$. If $\omega$ is some tensor field on $M$ then $\nabla \omega$ is not 
tensorial in $\omega$, but $(\nabla-D) \omega$ is. Let $\Gamma_{j k}^{i}=\Gamma_{j k}^{i}(t)$ be the Christoffel symbol for $g(t)$, so that

$$
\Gamma_{j k}^{i}=\frac{g^{i \ell}}{2}\left(\partial_{j} g_{k \ell}+\partial_{k} g_{j \ell}-\partial_{\ell} g_{j k}\right), \quad \nabla_{\partial_{j}} \partial_{k}=\Gamma_{j k}^{i} \partial_{i} .
$$

Set

$$
\bar{\Gamma}_{j k}^{i}=\Gamma_{j k}^{i}(t)-\Gamma_{j k}^{i}(0)
$$

Then

$$
\bar{\Gamma}_{j k}^{i}=\frac{g^{i \ell}}{2}\left(D_{j} g_{k \ell}+D_{k} g_{j \ell}-D_{\ell} g_{j k}\right) .
$$

This can be verified at each point by working in normal coordinates for the metric $g_{0}$.

Now the curvature tensor is

$$
R_{i j k}^{\ell}=\partial_{i} \Gamma_{j k}^{\ell}-\partial_{j} \Gamma_{i k}^{\ell}+\Gamma_{i p}^{\ell} \Gamma_{j k}^{p}-\Gamma_{j p}^{\ell} \Gamma_{i k}^{p},
$$

and

$$
\bar{R}_{i j k}^{\ell}=R_{i j k}^{\ell}(t)-R_{i j k}^{\ell}(0) .
$$

Again working in normal coordinates for $g_{0}$, we find that

$$
\bar{R}_{i j k}^{\ell}=D_{i} \bar{\Gamma}_{j k}^{\ell}-D_{j} \bar{\Gamma}_{i k}^{\ell}+\bar{\Gamma}_{i p}^{\ell} \bar{\Gamma}_{j k}^{p}-\bar{\Gamma}_{j p}^{\ell} \bar{\Gamma}_{i k}^{p} .
$$

This enables us to compute the first and second variations of the Ricci curvature. Indeed, we write

$$
\dot{g}_{i j}(0)=h_{i j}, \quad \ddot{g}_{i j}(0)=w_{i j}, \quad\left(g^{i j}\right)^{\cdot \cdot}(0)=z^{i j},
$$

so that

$$
z^{i j}=-w^{i j}+2 h^{i k} h_{k}^{j}
$$

Then

$$
\begin{aligned}
\dot{\Gamma}_{j k}^{i}(0) & =\frac{1}{2}\left(D_{j} h_{k}^{i}+D_{k} h_{j}^{i}-D^{i} h_{j k}\right) \\
\dot{\Gamma}_{i k}^{i}(0) & =\frac{1}{2} D_{k} h_{i}^{i}, \\
\ddot{\Gamma}_{j k}^{i}(0) & =\frac{1}{2}\left(D_{j} w_{k}^{i}+D_{k} w_{j}^{i}-D^{i} w_{j k}\right)-h^{i \ell}\left(D_{j} h_{k \ell}+D_{k} h_{j \ell}-D_{\ell} h_{j k}\right), \\
\ddot{\Gamma}_{i k}^{i}(0) & =\frac{1}{2} D_{k} w_{i}^{i}-h^{i \ell} D_{k} h_{i \ell}, \\
\dot{R}_{i j k}^{i}(0) & =D_{i} \dot{\Gamma}_{j k}^{i}-D_{j} \dot{\Gamma}_{i k}^{i}, \\
\ddot{R}_{i j k}^{i}(0) & =D_{i} \ddot{\Gamma}_{j k}^{i}-D_{j} \ddot{\Gamma}_{i k}^{i}+2\left(\dot{\Gamma}_{i \ell}^{i} \dot{\Gamma}_{j k}^{\ell}-\dot{\Gamma}_{j \ell}^{i} \dot{\Gamma}_{i k}^{\ell}\right)
\end{aligned}
$$

so that

$\dot{S}=\partial_{t}\left(g^{j k} R_{j k}\right)=-h^{j k} R_{j k}+g^{j k} \dot{R}_{j k}=-h^{j k} R_{j k}+D_{j} D_{k} h^{j k}-D_{j} D^{j} h_{k}^{k}$. 
This proves Lemma 3.2.1. To calculate the second variation of the scalar curvature we restrict to the case $h_{i}^{i}=0$ and $D_{j} h_{k}^{j}=0$. In this case $\dot{\Gamma}_{i k}^{i}=0$ and $\ddot{\Gamma}_{i k}^{i}=0$, so that

$$
\begin{aligned}
\ddot{S}=\partial_{t}^{2}\left(g^{j k} R_{j k}\right)= & z^{j k} R_{j k}-2 h^{j k} \dot{R}_{j k}+g^{j k} \ddot{R}_{j k} \\
= & z^{j k} R_{j k}-2 h^{j k}\left(D_{i} \dot{\Gamma}_{j k}^{i}-D_{j} \dot{\Gamma}_{i k}^{i}\right) \\
& \quad+g^{j k}\left(D_{i} \ddot{\Gamma}_{j k}^{i}-D_{j} \ddot{\Gamma}_{i k}^{i}+2 \dot{\Gamma}_{i \ell}^{i} \dot{\Gamma}_{j k}^{\ell}-2 \dot{\Gamma}_{j \ell}^{i} \dot{\Gamma}_{i k}^{\ell}\right) \\
= & z^{j k} R_{j k}-2 h^{j k} D_{i} \dot{\Gamma}_{j k}^{i}+g^{j k}\left(D_{i} \ddot{\Gamma}_{j k}^{i}-2 \dot{\Gamma}_{j \ell}^{i} \dot{\Gamma}_{i k}^{\ell}\right) .
\end{aligned}
$$

Now

$$
\begin{aligned}
-2 h^{j k} D_{i} \dot{\Gamma}_{j k}^{i}= & -2 h^{j k} D_{i} D_{j} h_{k}^{i}+h^{j k} D_{i} D^{i} h_{j k}, \\
g^{j k} D_{i} \ddot{\Gamma}_{j k}^{i}= & D_{i} D_{j} w^{i j}-\frac{1}{2} D_{i} D^{i} w_{j}^{j} \\
= & -D_{i} D_{j} z^{i j}+2\left(D_{i} D_{j} h^{i \ell}\right) h_{\ell}^{j} \\
& +2\left(D^{j} h^{i \ell}\right) D_{i} h_{\ell}^{j}-\frac{1}{2} D_{i} D^{i}\left(h_{j \ell} h^{j \ell}\right), \\
-2 g^{j k} \dot{\Gamma}_{j \ell}^{i} \dot{\Gamma}_{i k}^{\ell}= & -\frac{1}{2}\left(D_{j} h_{\ell}^{i}+D_{\ell} h_{j}^{i}-D^{i} h_{j \ell}\right)\left(D_{i} h^{j \ell}+D^{j} h_{i}^{\ell}-D^{\ell} h_{i}^{j}\right) \\
= & \frac{1}{2} D^{i} h^{j \ell} D_{i} h_{j \ell}-\left(D^{j} h^{i \ell}\right) D_{i} h_{j \ell} .
\end{aligned}
$$

Substituting this into (3.4.2) gives

$$
\ddot{S}=z^{j k} R_{j k}-D_{i} D_{j} z^{i j}+\left(D^{j} h_{i \ell}\right) D_{i} h_{j \ell}-\frac{1}{2}\left(D^{i} h^{j \ell}\right) D_{i} h_{j \ell} .
$$

\section{Critical points have finite index}

In this section we prove Theorem 1.3.1. As usual let $g_{0}$ be a metric on the $n$-dimensional closed compact manifold $M$ with $n$ odd, write tensors with respect to a $g_{0}$-orthonormal frame for $T M$ and write $d \mu_{0}$ for the volume element for $g_{0}$ normalized so that $\mu_{0}(M)=1$. Write $S_{0}^{2} T^{*} M$ for the bundle of symmetric $(0,2)$-tensors on $M$ which are trace-free relative to $g_{0}$.

Lemma 4.1.1. (a) The operators $P, Q: C^{\infty}(M) \rightarrow C^{\infty}(M)$ defined by

$$
\begin{aligned}
& (P \phi)(x) d \mu_{0}(x)=\operatorname{KERD}\left(\bar{\Delta}_{0}^{-1} \phi \bar{\Delta}_{0}^{-1}, x\right), \\
& (Q \phi)(x) d \mu_{0}(x)=\operatorname{KERD}\left(L^{-1} \phi L^{-1}, x\right),
\end{aligned}
$$

are pseudodifferential operators of order $n-4 . P$ and $Q$ have the same principal symbol $p$, where $(-1)^{(n+1) / 2} p(x, \xi)>0$. 
(b) The operator $R_{0}: C^{\infty}\left(S_{0}^{2} T^{*} M\right) \rightarrow C^{\infty}(M)$ defined by

$$
\left(R_{0} A\right)(x) d \mu_{0}(x)=\operatorname{KERD}\left(\bar{\Delta}_{0}^{-1} D_{k \ell}^{2} A_{k \ell} \bar{\Delta}_{0}^{-1}, x\right)
$$

is a pseudodifferential operator of order $n-2$, and $R_{0}$ has the same principal symbol as the operator

$$
R_{1}=C^{(n)} \Delta_{0}^{(n-4) / 2} D_{k \ell}^{2},
$$

where $C^{(n)}$ is a constant, and $D_{k \ell}^{2}$ denotes the divergence operator $A_{k \ell} \rightarrow$ $D_{k \ell}^{2} A_{k \ell}$.

(c) The operators $U, V: C^{\infty}\left(S_{0}^{2} T^{*} M\right) \rightarrow C^{\infty}\left(S_{0}^{2} T^{*} M\right)$ defined by

$$
\begin{aligned}
& (U A(x))_{k \ell} d \mu_{0}(x)=\operatorname{KERD}\left(\bar{\Delta}_{0}^{-1} D_{i j}^{2} A_{i j} \bar{\Delta}_{0}^{-1} D_{k \ell}^{2}, x\right), \\
& (V A(x))_{k \ell} d \mu_{0}(x)=\operatorname{KERD}\left(L_{0}^{-1} D_{i j}^{2} A_{i j} L_{0}^{-1} D_{k \ell}^{2}, x\right),
\end{aligned}
$$

are elliptic of order $n$. $U$ and $V$ have the same principal symbol $u$, where $(-1)^{(n+1) / 2} u(x, \xi)>0$.

Remark. In general, the homogeneous terms in the symbol expansions of $P, Q, R_{0}, U, V$ down to but not including the term of order zero, can be explicitly computed from $g_{0}, \phi, A_{i j}$. To obtain terms of order $|\xi|^{0}$ and lower involves computing the Taylor expansion at the diagonal of the regular part of the Green function for $\bar{\Delta}_{0}$ or $L$. In Section 5 , we will consider the case when $g_{0}$ is the standard metric on $S^{n}$ and we will compute $U$ explicitly for $n=3$ and $V$ explicitly for all odd $n$.

Proof of Theorem 1.3.1. We will see that there exists an order $n$ pseudodifferential operator $Y: C^{\infty}\left(S^{2} T^{*} M\right) \rightarrow C^{\infty}\left(S^{2} T^{*} M\right)$ such that whenever

$$
h_{i j}=2 \phi \delta_{i j}+A_{i j}, \quad \text { with } \quad A_{i i}=0, \quad A_{i j, j}=0,
$$

we have

$$
\operatorname{Hess} F(h, h)=\int_{M} h_{i j}(Y h)_{i j} .
$$

Moreover, the principal symbol $y$ of $Y$ satisfies $(-1)^{(n+1) / 2} y(x, \xi)>0$, and hence by elliptic theory $Y$ has at most finitely many nonnegative eigenvalues if $n=4 m+3$ and finitely many nonpositive eigenvalues in $n=4 m+1$.

To see that (4.1.2) holds, we must consider the terms in (1.3.10). The two main terms are

$$
-\left(\frac{n-2}{2}\right)^{2} \operatorname{TR} \phi_{i i} \bar{\Delta}_{0}^{-1} \phi_{k k} \bar{\Delta}_{0}^{-1}=\int_{M} \phi\left(\Delta_{0} P \Delta_{0} \phi\right) d \mu_{0},
$$

and

$$
-\operatorname{TR} D_{i} A_{i j} D_{j} \bar{\Delta}_{0}^{-1} D_{k} A_{k \ell} D_{\ell} \bar{\Delta}_{0}^{-1}=\int_{M} A_{i j}(U A)_{i j} d \mu_{0}
$$


Here we have used Lemma 4.1.1. Now the map

$$
h \rightarrow(\phi, A), \quad \text { where } \quad h_{i j}=2 \phi \delta_{i j}+A_{i j}, \quad A_{i i}=0,
$$

gives rise to the splitting

$$
C^{\infty}\left(S^{2} T^{*} M\right)=L M \oplus C^{\infty}\left(S_{0}^{2} T^{*} M\right)
$$

where $L M$ is the trivial line bundle over $M$. We can use this splitting to extend $P$ and $U$ to operators on $C^{\infty}\left(S^{2} T^{*} M\right)$ in the natural way, so that the sum of (4.1.3) and (4.1.4) is

$$
\int_{M} \phi\left(\Delta_{0} P \Delta_{0} \phi\right)+\int_{M} A_{i j}(U A)_{i j}=\int_{M} h_{i j}\left(Y_{0} h\right)_{i j} d \mu_{0}
$$

where $Y_{0}$ is an elliptic pseudodifferential operator on $C^{\infty}\left(S^{2} T^{*} M\right)$ of order $n$ and its principal symbol $y$ in normal coordinates satisfies

$$
y_{i j k \ell}(x, \xi) h_{i j} h_{k \ell}=-(n-2)^{2} p(x, \xi)|\xi|^{4}(\phi(x))^{2}-u_{i j k \ell}(x, \xi) A_{i j} A_{k \ell},
$$

and $(-1)^{(n-1) / 2} y(x, \xi)>0$. The other terms in (1.3.10) correspond to operators of lower order. In particular when $A_{i j, j}=0$ we see that $R_{1} A=0$ and so setting $R=R_{0}-R_{1}$ we have $R_{0} A=R A$. But $R$ is a pseudodifferential operator of order $n-3$. The cross term in (1.3.10) is

$$
-(n-2) \operatorname{TR} \phi_{i i} \bar{\Delta}_{0}^{-1} D_{k} A_{k \ell} D_{\ell} \bar{\Delta}_{0}^{-1}=(n-2) \int_{M} \phi \Delta_{0} R A d \mu_{0} .
$$

The operator $\Delta_{0} R$ has order $n-1$ which is less than the order of $Y_{0}$. We conclude that (4.1.2) holds where $Y$ has the same principal symbol as $Y_{0}$.

Similarly by Lemma 4.1.1 and Lemma 3.4.1,

$$
\text { Hess log } \operatorname{det} L(A, A)=\int_{M} A_{i j}(Z A)_{i j}
$$

where $Z$ is an elliptic pseudodifferential operator of order $n$, whose principal symbol $z=-u$, and so $Z$ has at most finitely many nonnegative eigenvalues if $n=3 \bmod 4$ and finitely many nonpositive eigenvalues in $n=1 \bmod 4$.

Proof of Lemma 4.1.1. We apply Theorem 1.2.6. Work in normal coordinates $w$ about the point $x \in M$ and extend the orthonormal frame $\partial_{w_{1}}, \ldots, \partial_{w_{n}}$ at $x$ smoothly to a local orthonormal frame for $T M$, thus giving a local trivialization of $S_{0}^{2} T^{*} M$. The principal symbol of $D_{k \ell}^{2}$ at $x$ is $-\xi_{k} \xi_{\ell}$, and the principal symbol of $\bar{\Delta}^{-1}$ and $L^{-1}$ at $x$ is $|\xi|^{-2}$.

The Fourier transform of $|w|^{s}$ on $\mathbb{R}^{n}$ is

$$
C(s)|\xi|^{-n-s}, \quad C(s)=C_{n}(s)=\frac{\pi^{n / 2} 2^{n+s} \Gamma\left(\frac{n+s}{2}\right)}{\Gamma(-s / 2)}
$$

when $s \notin\{-n, 2-n, \ldots\}$; see [Ta]. Set

$$
C^{\prime}=\frac{\Gamma(n / 2)}{2^{n+3} \pi^{(n / 2)-1}(n+1) \Gamma(n)}>0 .
$$


(a) By Theorem 1.2.6, $P$ and $Q$ are pseudodifferential operators and the principal symbol of each is

$$
\begin{aligned}
p(x, \xi) & =\left(\left(|\xi|^{-2}\right)^{\vee}\left(|\xi|^{-2}\right)^{\vee}\right)^{\wedge}=\left(\frac{1}{2 \pi}\right)^{2 n}(C(-2))^{2}\left(|w|^{2-n}|w|^{2-n}\right)^{\wedge} \\
& =\left(\frac{1}{2 \pi}\right)^{2 n}(C(-2))^{2} C(4-2 n)|\xi|^{n-4} \\
& =(-1)^{(n+1) / 2} 16(n-1)(n+1) C^{\prime}|\xi|^{n-4} .
\end{aligned}
$$

(b) By Theorem 1.2.6, $R_{0}$ is a pseudodifferential operator. Its principal symbol,

$$
r(x, \xi): S_{0}^{2} T_{x}^{*} M \rightarrow \mathbb{C}
$$

can be written as a symmetric matrix $r_{k \ell}(x, \xi)$ so that

$$
r(x, \xi) A=r_{k \ell}(x, \xi) A_{k \ell} .
$$

Since $A$ is trace-free, we can calculate $r_{k \ell}(x, \xi)$ modulo terms of the form $u(\xi) \delta_{k \ell}$.

$$
\begin{aligned}
r_{k \ell}(x, \xi) & =-\left(\left(|\xi|^{-2}\right)^{\vee}\left(\xi_{k} \xi_{\ell}|\xi|^{-2}\right)^{\vee}\right)^{\wedge} \\
& =\left(\frac{1}{2 \pi}\right)^{2 n}(C(-2))^{2}\left(|w|^{2-n} \frac{\partial^{2}}{\partial w_{k} \partial w_{\ell}}|w|^{2-n}\right)^{\wedge} \\
& =\left(\frac{1}{2 \pi}\right)^{2 n}(C(-2))^{2} n(n-2)\left(|w|^{-2 n} w_{k} w_{\ell}\right)^{\wedge} \\
& =-\left(\frac{1}{2 \pi}\right)^{2 n}(C(-2))^{2} C(-2 n) n(n-2) \frac{\partial^{2}}{\partial \xi_{k} \partial \xi_{\ell}}|\xi|^{n} \\
& =-\left(\frac{1}{2 \pi}\right)^{2 n}(C(-2))^{2} C(-2 n) n^{2}(n-2)^{2}|\xi|^{n-4} \xi_{k} \xi_{\ell} .
\end{aligned}
$$

Clearly the operator

$$
R_{1}=-\left(\frac{1}{2 \pi}\right)^{2 n}(C(-2))^{2} C(-2 n) n^{2}(n-2)^{2} \Delta_{0}^{(n-4) / 2} D_{i} D_{j}
$$

has the same principal symbol as $R_{0}$.

(c) Finally, by Theorem 1.2.6, $U$ and $V$ are pseudodifferential operators and their principal symbol $u$ can be written as a tensor $u_{i j k \ell}$ symmetric in $i$ and $j$ and in $k$ and $\ell$, with

$$
(u(x, \xi) A)_{i j}=u_{i j k \ell}(x, \xi) A_{k \ell},
$$


and this matrix can be calculated modulo terms of the form $\delta_{i j} v(\xi)+w(\xi) \delta_{k \ell}$.

$$
\begin{aligned}
u_{i j k \ell} & =\left(\left(|\xi|^{-2} \xi_{i} \xi_{j}\right)^{\vee}\left(\xi_{k} \xi_{\ell}|\xi|^{-2}\right)^{\vee}\right)^{\wedge} \\
& =\left(\frac{1}{2 \pi}\right)^{2 n}(C(-2))^{2}\left(\left(\frac{\partial^{2}}{\partial w_{i} \partial w_{j}}|w|^{2-n}\right)\left(\frac{\partial^{2}}{\partial w_{k} \partial w_{\ell}}|w|^{2-n}\right)\right)^{\wedge} \\
& =\left(\frac{1}{2 \pi}\right)^{2 n}(C(-2))^{2} n^{2}(n-2)^{2}\left(|w|^{-4-2 n} w_{i} w_{j} w_{k} w_{\ell}\right)^{\wedge} \\
& =\left(\frac{1}{2 \pi}\right)^{2 n}(C(-2))^{2} C(-2 n-4) n^{2}(n-2)^{2} \frac{\partial^{4}}{\partial \xi_{i} \partial \xi_{j} \partial \xi_{k} \partial \xi_{\ell}}|\xi|^{n+4} \\
& =\frac{(-1)^{(n+1) / 2} C^{\prime}}{(n+2)(n+4)} \frac{\partial^{4}}{\partial \xi_{i} \partial \xi_{j} \partial \xi_{k} \partial \xi_{\ell}}|\xi|^{n+4}=(-1)^{(n+1) / 2} C^{\prime} \tilde{u}_{i j k \ell}
\end{aligned}
$$

where

$$
\begin{aligned}
\tilde{u}_{i j k \ell}=n(n-2)|\xi|^{n-4} \xi_{i} \xi_{j} \xi_{k} \xi_{\ell} & \\
& +n|\xi|^{n-2}\left(\xi_{i} \xi_{k} \delta_{j \ell}+\xi_{i} \xi_{\ell} \delta_{j k}+\xi_{j} \xi_{\ell} \delta_{i k}\right. \\
& \left.+\xi_{j} \xi_{k} \delta_{i \ell}\right) \\
& +|\xi|^{n}\left(\delta_{i k} \delta_{j \ell}+\delta_{j k} \delta_{i \ell}\right) .
\end{aligned}
$$

The symbol $\tilde{u}$ is clearly elliptic and positive. Indeed dropping the summation convention, we have

$$
\begin{aligned}
\sum_{i, j, k, \ell} A_{i j} \tilde{u}_{i j k \ell} A_{k \ell}= & n(n-2)\left(\sum_{i, j} A_{i j} \xi_{i} \xi_{j}\right)^{2}|\xi|^{n-4} \\
& +4 n \sum_{j}\left(\sum_{i} A_{i j} \xi_{i}\right)^{2}|\xi|^{n-2}+2 \sum_{i, j}\left(A_{i j}\right)^{2}|\xi|^{n}
\end{aligned}
$$

\section{Standard spheres which are extremal}

Formulas for the Hessian of the functionals $F=\log \operatorname{det}^{\prime} \Delta-(2 / n) \log V$ and $\log \operatorname{det} L$ at general critical points were proved in Sections 3.3 and 3.4. In Section 4 it was shown that the critical points always have finite index. In this section we evaluate explicitly the formula for Hess $F$ on the 3 -sphere and for Hess $\log \operatorname{det} L$ on all odd dimensional spheres, to show that these spheres are local extremals. By contrast, in Section 6 we will see that the $(4 m+3)$-sphere with $m=1,2, \ldots$, is a saddle point for $F$.

In Section 5.1, we develop the general theory to compute the Hessian of the log determinant functionals on the standard spheres, and in Sections 5.2 
and 5.3 we apply this theory to $F$ and $\log \operatorname{det} L$ respectively. Throughout we write $S S^{n}$ for the unit sphere bundle of $S^{n}, g_{0}$ for the standard metric on $S^{n}$, $d \sigma_{n}$ for the standard volume form, $V_{n}$ for the volume $\sigma_{n}\left(S^{n}\right)$ and $d \mu_{n}$ for the normalized volume form $d \sigma_{n} / V_{n}$. We write $S_{0}^{2} T^{*} M$ for the bundle of symmetric $(0,2)$ tensors on $M$ which are trace-free relative to $g_{0}$, and the components of all tensors are given with respect to a $g_{0}$-orthonormal frame.

\subsection{Fourier series for singular kernels.}

Lemma 5.1.1. Let $P, Q: C^{\infty}\left(S^{n}\right) \rightarrow C^{\infty}\left(S^{n}\right)$ be odd class pseudodifferential operators which are functions of the Laplacian on the standard $n$-sphere, and suppose $A \in C^{\infty}\left(S_{0}^{2} T^{*} S^{n}\right)$ and $A_{i j, j}=0$. Then

$$
\operatorname{KERD}\left(P A_{i j} D_{i j}^{2} Q, x\right)=0 .
$$

Proof. Write $\mathbb{T}$ for the circle $\mathbb{R} /(2 \pi \mathbb{Z})$. It is convenient to introduce some spaces of functions on $\mathbb{T} \backslash 0$.

$$
\begin{aligned}
\mathcal{F} & =\left\{f \in C^{\infty}(\mathbb{T} \backslash 0): f(x)=f(-x)\right\} . \\
\mathcal{F}_{0} & =\{f \in \mathcal{F}: f(\pi)=0\} .
\end{aligned}
$$

Define $T: \mathcal{F} \rightarrow \mathcal{F}_{0}$ by

$$
T f=f^{\prime \prime}(r)-\frac{\cos r}{\sin r} f^{\prime}(r)=\sin r \frac{d}{d r} \frac{1}{\sin r} \frac{d f}{d r} .
$$

The right inverse $T^{-1}$ is given by

$T^{-1} f:=I\left(\sin r I\left(\frac{f}{\sin r}\right)\right), \quad$ where $\operatorname{If}(x)=\int_{\pi}^{x} f(t) d t, \quad x \in(0,2 \pi)$.

Write $r=r(x, y)$ for the distance between points $x$ and $y$ measured on the standard $n$-sphere. If $P$ and $Q$ are functions of the Laplacian then we can define $\Psi, \Phi \in \mathcal{F}$ by

$$
\Phi(r)=K(P, x, y), \quad \Psi(r)=K(Q, x, y), \quad 0<r \leq \pi,
$$

and extend $\Phi$ and $\Psi$ to functions in $\mathcal{F}$. On the standard sphere, $D_{y, i j}^{2} \cos r=$ $-\delta_{i j} \cos r$. Here, the subscript $y$ indicates that we are taking the partial covariant derivative with respect to $y$. We have

$$
D_{y, i j}^{2} \Psi(r)=\left(\Psi^{\prime \prime}(r)-\frac{\cos r}{\sin r} \Psi^{\prime}(r)\right) r_{y, i} r_{y, j}+\frac{\cos r}{\sin r} \Psi^{\prime}(r) \delta_{i j} .
$$

Write $a(x, y)=r_{y, i} r_{y, j} A_{i j}(y)$. Since $A_{i i}=0$, we get

$$
\begin{aligned}
K\left(A_{i j} D_{i j}^{2} Q, y, x\right) & =A_{i j}(y) D_{y, i j}^{2} K(Q, y, x) \\
& =A_{i j}(y)(T \Psi(r)) r_{y, i} r_{y, j}=T \Psi(r) a(x, y) .
\end{aligned}
$$


First we consider the case when $P$ and $Q$ are pseudodifferential operators which are functions of the standard Laplacian, ord $P<-n$ and $\operatorname{ord} Q<-n-2$. For the following argument we do not need to assume that $P$ and $Q$ are odd class or integral order.

$$
\begin{aligned}
K & \left(P A_{i j} D_{i j}^{2} Q, x, x\right) \\
& =\int_{S^{n}} K(P, x, y) K\left(A_{i j} D_{i j}^{2} Q, y, x\right) d \mu_{n}(y) \\
& =\frac{1}{V_{n}} \int_{S^{n}} \Phi(r) a(x, y)(T \Psi)(r) d \sigma_{n}(y) \\
& =\frac{1}{V_{n}} \int_{r=0}^{\pi} \int_{v \in S S_{x}^{n}} a\left(x, \exp _{x}(r v)\right) \Phi(r)(T \Psi)(r) \sin ^{n-1} r d r d \sigma_{n-1}(v) \\
& =\frac{1}{V_{n}} \int_{r=0}^{\pi} \int_{v \in S S_{x}^{n}} a\left(x, \exp _{x}(r v)\right) T T^{-1}\left(\Phi(r)(T \Psi)(r) \sin ^{n-1} r\right) d r d \sigma_{n-1}(v) \\
& =\int_{S^{n}} A_{i j}(y) D_{y, i j}^{2} T^{-1}\left(\Phi(r)(T \Psi)(r) \sin ^{n-1} r\right) d \mu_{n}(y) \\
& =\int_{S^{n}}\left(D_{y, i j}^{2} A_{i j}(y)\right) T^{-1}\left(\Phi(r)(T \Psi)(r) \sin ^{n-1} r\right) d \mu_{n}(y)=0 .
\end{aligned}
$$

The orders of $P$ and $Q$ were chosen low enough so that all distributions involved are bounded functions and integration by parts is valid. The calculation fails when $\operatorname{ord} P$ and $\operatorname{ord} Q$ are large, but the lemma is easily obtained by setting

$$
P_{z}=P(I+\Delta)^{z / 4}, \quad Q_{z}=Q(I+\Delta)^{z / 4} ;
$$

thus applying the lemma when $\Re z \ll 0$ gives $K\left(P_{z} A_{i j} D_{i j}^{2} Q_{z}, x, x\right)=0$. Hence by analytic continuation, $\operatorname{KERD}\left(P_{0} A_{i j} D_{i j}^{2} Q_{0}, x\right)=0$.

We will now derive a formula for $\operatorname{TR}\left(A_{i j} D_{i j}^{2} P B_{k \ell} D_{k \ell}^{2} Q\right)$ when $A, B \in$ $C^{\infty}\left(S_{0}^{2} T^{*} S^{n}\right)$.

For $(x, v) \in S S^{n}$ and $r \in \mathbb{R}$, let $\left(x_{r}, v_{r}\right)$ be the image of $(x, v)$ after flowing distance $r$ along the geodesic with initial point $x$ and initial direction $v$, so that $\left(x_{r}, v_{r}\right)=\left(\left.\exp \right|_{x}(r v),\left.\exp _{*}\right|_{(x, r v)}(v)\right)$. For a function $g$ on $\mathbb{T}$, define the Fourier coefficients of $g$ :

$$
\hat{g}_{k}=\frac{1}{2 \pi} \int_{0}^{2 \pi} g(r) e^{i k r} d r .
$$

This definition extends to distributions $g$ by duality. For a function $a(x, v)$ on $S S^{3}$, define

$$
\hat{a}_{k}(x, v)=\frac{1}{2 \pi} \int_{0}^{2 \pi} a\left(x_{s}, v_{s}\right) e^{i k s} d r
$$


Lemma 5.1.2. Suppose that $n$ is odd and $P$ and $Q$ are odd class pseudodifferential operators on $S^{n}$ which are functions of the standard Laplacian. Suppose that $P, Q$ have order at most -2. Let $A, B \in C^{\infty}\left(S_{0}^{2} T^{*} S^{n}\right)$ and for $v \in T_{x}^{*} S^{n}$ write $a(x, v)=A_{i j}(x) v_{i} v_{j}, b(x, v)=B_{i j}(x) v_{i} v_{j}$. Then

$$
\begin{aligned}
\operatorname{TR}\left(A_{i j} D_{i j}^{2} P B_{k \ell} D_{k \ell}^{2} Q, x\right) \\
=\frac{\pi}{V_{n}^{2}} \int_{S S^{n}} \sum_{k=-\infty}^{\infty} \hat{f}_{k} \hat{a}_{k}(x, v) \hat{b}_{-k}(x, v) d \sigma_{n-1}(v) d \sigma_{n}(x)
\end{aligned}
$$

where $f(r)$ is the even distribution on $\mathbb{T}$ defined by

$$
f(r)=T \Phi(r) T \Psi(r) \sin ^{n-1} r, \quad 0<r<2 \pi,
$$

and regularized so that for $r$ close to zero, $f(r)$ is the sum of a polyhomogeneous distribution with regular parity and a bounded function. There is an asymptotic expansion for $\hat{f}_{k}$ :

$$
\hat{f}_{k} \sim c_{n}|k|^{n}+c_{n-2}|k|^{n-2}+\ldots+c_{1}|k|+o(1), \quad|k| \rightarrow \infty
$$

Proof. First, we consider the case when $P$ and $Q$ are pseudodifferential operators which are functions of the standard Laplacian and $\operatorname{ord} P, \operatorname{ord} Q<-2$, $\operatorname{ord} P+\operatorname{ord} Q<-n-4$. For the following argument we do not need to assume that $P$ and $Q$ are odd class or integral order. The function $f(r)$ in (5.1.6) is integrable and

$$
\begin{aligned}
K & \left(A_{i j} D_{i j}^{2} P B_{k \ell} D_{k \ell}^{2} Q, x, x\right) \\
& =\frac{1}{V_{n}} \int_{S^{n}} A_{i j}(x)\left(D_{x, i j}^{2} \Phi(r)\right) B_{k \ell}(y)\left(D_{y, k \ell}^{2} \Psi(r)\right) d \sigma_{n}(y) \\
& =\frac{1}{V_{n}} \int_{S^{n}} A_{i j}(x) r_{x ; i} r_{x ; j} B_{k \ell}(y) r_{y ; k} r_{y ; l} T \Phi(r) T \Psi(r) d \sigma_{n}(y) \\
& =\frac{1}{2 V_{n}} \int_{S S_{x}^{3}} \int_{0}^{2 \pi} a(x, v) b\left(x_{r}, v_{r}\right) T \Phi(r) T \Psi(r) \sin ^{n-1} r d r d \sigma_{n-1}(v) \\
& =\frac{1}{2 V_{n}} \int_{S S_{x}^{3}} \int_{0}^{2 \pi} f(r) a(x, v) b\left(x_{r}, v_{r}\right) d r d \sigma_{n-1}(v) .
\end{aligned}
$$


Integrating over $x \in S^{n}$, we get

$$
\begin{aligned}
\operatorname{trace} & \left(A_{i j} D_{i j}^{2} P B_{k \ell} D_{k \ell}^{2} Q, x, x\right) \\
= & \frac{1}{2 V_{n}^{2}} \int_{S S^{3}} \int_{0}^{2 \pi} f(r) a(x, v) b\left(x_{r}, v_{r}\right) d r d \sigma_{n-1}(v) d \sigma_{n}(x) \\
= & \frac{1}{4 \pi V_{n}^{2}} \int_{S S^{3}} \int_{0}^{2 \pi} \int_{0}^{2 \pi} a\left(x_{s}, v_{s}\right) b\left(x_{s+r}, v_{s+r}\right) f(r) d s d r d \sigma_{n-1}(v) d \sigma_{n}(x) \\
= & \frac{\pi}{V_{n}^{2}} \int_{S S^{3}} \sum_{k=-\infty}^{\infty} \hat{f}_{k} \hat{a}_{k}(x, v) \hat{b}_{-k}(x, v) d \sigma_{n-1}(v) d \sigma_{n}(x) .
\end{aligned}
$$

Here, we have used the fact that the measure $d \sigma_{n-1}(v) d \sigma_{n}(x)$ is invariant under the geodesic flow $(x, v) \rightarrow\left(x_{s}, v_{s}\right)$.

To establish the general case we may suppose without loss of generality that $P$ and $Q$ have order -2 , form the analytic family $P_{z}=P(I+\Delta)^{z / 2}$ and set $\Phi_{z}(r)=K\left(P_{z}, x, y\right)$ and $f_{z}(r)=T \Phi_{z}(r) T \Psi(r) \sin ^{n-1} r$. Then there exist constants $p_{j}(z), u_{j}(z), c_{j}(z), \tilde{c}_{j}(z)$ depending analytically on $z$ when $\Re z<2$, such that as $r \downarrow 0$,

$$
\begin{aligned}
\Phi_{z}(r) \sim\left(p_{2-n}(z) r^{2-n-z}+p_{4-n}(z) r^{4-n-z}\right. & +\ldots) \\
& +\left(p_{0}(z)+p_{2}(z) r^{2}+\ldots\right), \\
& +\ldots) \\
T \Phi_{z}(r) \sim\left(t_{-n}(z) r^{-n-z}+t_{2-n}(z) r^{2-n-z}\right. & +\left(t_{2}(z) r^{2}+t_{4}(z) r^{4}+\ldots\right), \\
T \Psi(r) \sim\left(u_{-n} r^{-n}+u_{2-n} r^{2-n}+\ldots\right)+ & \left(u_{2} r^{2}+u_{4} r^{4}+\ldots\right),
\end{aligned}
$$

so that

$$
\begin{aligned}
& f_{z}(r) \sim\left(c_{-1-n}(z) r^{-1-n-z}+c_{1-n}(z) r^{1-n-z}+\ldots\right) \\
& \quad+\left(c_{1}(z) r^{1-z}+c_{3}(z) r^{3-z}+\ldots\right) \\
& \quad+\left(\tilde{c}_{0}(z)+\tilde{c}_{1}(z) r+\tilde{c}_{2}(z) r^{2}+\ldots\right) .
\end{aligned}
$$

The terms $c_{j}(z) r^{j-z}$ with even $j<0$ analytically continue to $z=0$ to give regular parity distributions, and

$$
f_{z}(r)-\left(c_{-1-n}(z) r^{-1-n-z}+c_{1-n}(z) r^{1-n-z}+\ldots+c_{-1}(z) r^{-1-z}\right)
$$

analytically continues to $z=0$ to give a bounded function of $r$. Hence as a distribution, $f_{z}(r)$ analytically continues to $z=0$ to give a regular parity distribution in $r$ plus an integrable function of $r$. The Fourier coefficients of the function $f_{0}$ clearly satisfy (5.1.7). Equation (5.1.8) holds when $z<<0$, and extends in the distributional sense to $z=0$. 
Finally we outline the procedure to calculate $\hat{f}_{k}$ from the function $f$.

Definition 5.1.3. If $f \in \mathcal{F}$, we say $\alpha_{k}$ are Fourier coefficients for $f$ if the sequence $\alpha_{k}$ is tempered; that is, for some $N$,

$$
\left|\alpha_{k}\right| \leq C\left(1+|k|^{N}\right),
$$

and the distribution

$$
\sum_{k} \alpha_{k} e^{-i k r}
$$

is equal to the function $f(r)$ on $\mathbb{T}^{n} \backslash 0$.

Lemma 5.1.4. Suppose $f$ is a function in $\mathcal{F}$ and $\alpha_{k}, \gamma_{k}$ are two sets of Fourier coefficients for $f$. Then there exists a polynomial $p(x)$ such that

$$
\alpha_{k}=\gamma_{k}+p(k) \text {. }
$$

Our strategy to compute the Fourier coefficients of the distribution $f$ in (5.1.6) will be to compute Fourier coefficients $\gamma_{k}$ for the restriction of $f$ to $\mathbb{T} \backslash 0$, and then to adjust these by adding on a polynomial $p(k)$ to get coefficients $\alpha_{k}$ with the asymptotics given by (5.1.7). These are then necessarily the Fourier coefficients of the distribution $f$.

To calculate Fourier coefficients of the function $f \in \mathcal{F}$, we look for a harmonic function $h(z)$ on the unit disc $\mathcal{D}=\{z \in \mathbb{C}:|z|<1\}$ which extends continuously to $\overline{\mathcal{D}} \backslash 1$ and equals $f$ on the circle in the sense that

$$
f(r)=h\left(e^{i r}\right), \quad r \in \mathbb{T} \backslash 0 .
$$

LEMmA 5.1.5. If (5.1.10) holds and

$$
h(z)=\alpha_{0}+\sum_{k=1}^{\infty}\left(\alpha_{k} z^{k}+\alpha_{-k} \bar{z}^{k}\right), \quad|z|<1,
$$

where $\alpha_{k}$ are tempered, then $\alpha_{k}$ are Fourier coefficients for $f$.

We omit the proofs of Lemmas 5.1.4 and 5.1.5. It is convenient to write

$$
f \sim \alpha_{0}+\sum_{k=1}^{\infty}\left(\alpha_{k} z^{k}+\alpha_{-k} \bar{z}^{k}\right)
$$

when (5.1.10) and (5.1.11) hold.

5.2. The Hessian of $F$ at $S^{3}$. Theorem 1.3.10 gives the Hessian of the function $F$ at a critical metric $g_{0}$. In dimension 3,

$$
\begin{aligned}
\text { Hess } & F\left(2 \phi g_{0}+A, 2 \phi g_{0}+A\right) \\
= & -\frac{1}{3} \int_{S^{3}} A_{i j} A_{i j} d \mu_{0}+\frac{5}{2} \int_{S^{3}} \bar{\phi}^{2} d \mu_{0}-\frac{1}{4} \operatorname{TR} \phi_{i i} \bar{\Delta}_{0}^{-1} \phi_{j j} \bar{\Delta}_{0}^{-1} \\
& -\operatorname{TR} A_{i j} D_{i j}^{2} \bar{\Delta}_{0}^{-1} \phi_{j j} \bar{\Delta}_{0}^{-1}-\operatorname{TR} A_{i j} D_{i j}^{2} \bar{\Delta}_{0}^{-1} A_{k \ell} D_{k \ell}^{2} \bar{\Delta}_{0}^{-1}
\end{aligned}
$$


where $A \in S_{0}^{2} T^{*} S^{n}$ with $A_{i j, j}=0$. Our aim is to show that this is strictly negative unless $A_{i j}=0$ and $\phi \delta_{i j} \in \operatorname{diff}\left(g_{0}\right)$. The term

$$
\operatorname{TR} A_{i j} D_{i j}^{2} \bar{\Delta}_{0}^{-1} \phi_{j j} \bar{\Delta}_{0}^{-1}
$$

vanishes by Lemma 5.1.1. Richardson [Ri] studied the case of conformal deformations and his results show that when $A_{i j}=0$, Hess $F \leq 0$ with strict inequality when $\phi \delta_{i j} \notin \operatorname{diff}\left(g_{0}\right)$. Hence we only need to understand the case $\phi=0$. For $n>0$, set

$$
\begin{aligned}
I(k) & = \begin{cases}1 & k \text { even, } \\
0 & k \text { odd. }\end{cases} \\
E(k) & =\sum_{j=1}^{k} \frac{I(k-j)}{j^{2}}+\frac{\pi^{2} I(k)}{12} .
\end{aligned}
$$

Lemma 5.2.1. For the standard metric on $S^{3}$, suppose that

$$
A \in C^{\infty}\left(S_{0}^{2} T^{*} S^{2}\right) \text {. }
$$

Write $a(x, v)=A_{i j}(x) v_{i} v_{j}$. Then

$$
\operatorname{TR} A_{i j} D_{i j}^{2} \bar{\Delta}_{0}^{-1} A_{k \ell} D_{k \ell}^{2} \bar{\Delta}_{0}^{-1}=\frac{1}{4 \pi^{3}} \int_{S S^{3}} \sum_{k=-\infty}^{\infty} \alpha_{k}\left|\hat{a}_{k}(x, v)\right|^{2} d \sigma_{2}(v) d \sigma_{3}(x)
$$

where $\alpha_{k}>0$ for all $k$, so that (5.2.3) is positive unless $A=0$.

Proof. Let $G(r)$ be Green's function where $r$ denotes the distance between $x$ and $y$; that is

$$
G(r)=K\left(\bar{\Delta}_{0}^{-1}, x, y\right)=\frac{(\pi-r) \cos r}{2 \sin r}+\frac{1}{2} .
$$

By Lemma 5.1.2, (5.2.3) holds where $\alpha_{k}$ are the Fourier coefficients of $f(r)=$ $(T G(r))^{2} \sin ^{2} r$. We will follow the strategy outlined below Lemma 5.1.4 to compute these Fourier coefficients. Now

$$
G^{\prime}(r)=\frac{-\cos r}{2 \sin r}+\frac{(r-\pi)}{2 \sin ^{2} r}
$$

and since

$$
G^{\prime \prime}(r)+\frac{2 \cos r}{\sin r} G^{\prime}(r)=1
$$

we see that

$$
\begin{aligned}
T G(r) & =G^{\prime \prime}(r)-\frac{\cos r}{\sin r} G^{\prime}(r)=1-\frac{3 \cos r}{\sin r} G^{\prime}(r) \\
& =1+\frac{3 \cos ^{2} r}{2 \sin ^{2} r}-\frac{3(r-\pi) \cos r}{2 \sin ^{3} r}=\frac{-3(r-\pi) \cos r}{2 \sin ^{3} r}+\frac{3}{2 \sin ^{2} r}-\frac{1}{2} .
\end{aligned}
$$


Hence

$$
\begin{aligned}
f(r)= & (T G(r))^{2} \sin ^{2} r=\left(\frac{-3(r-\pi) \cos r}{2 \sin ^{2} r}+\frac{3}{2 \sin r}-\frac{\sin r}{2}\right)^{2} \\
= & \frac{9(r-\pi)^{2} \cos ^{2} r}{4 \sin ^{4} r}-\frac{9(r-\pi) \cos r}{2 \sin ^{3} r}+\frac{9}{4 \sin ^{2} r} \\
& \quad+\frac{3(r-\pi) \cos r}{2 \sin r}-\frac{3}{2}+\frac{\sin ^{2} r}{4} \\
= & \frac{3}{8}\left(\frac{d^{2}}{d r^{2}}-2\right)\left(\frac{(r-\pi)^{2}}{\sin ^{2} r}-2 \frac{(r-\pi) \cos r}{\sin r}\right)-\frac{3}{2}+\frac{\sin ^{2} r}{4} \\
= & -\frac{3}{8}\left(\frac{d^{2}}{d r^{2}}-2\right) \frac{d}{d r} \frac{(r-\pi)^{2} \cos r}{\sin r}-\frac{3}{2}+\frac{\sin ^{2} r}{4} .
\end{aligned}
$$

We are now going to compute Fourier coefficients for $f$ restricted to $\mathbb{T} \backslash 0$. To do this we will find a harmonic function on the disc having $f$ as its boundary values. We rely on the simple fact that although products of harmonic functions are not in general harmonic, the product of two analytic functions or of two conjugate analytic functions is harmonic. Define

$$
p(z)=-i \frac{1+z^{2}}{1-z^{2}}=-i\left(1+2 \sum_{j=1}^{\infty} z^{2 j}\right), \quad h(z)=\frac{\pi^{2}}{6}+\sum_{k=1}^{\infty} \frac{2}{k^{2}} z^{k} .
$$

Setting $z=e^{i r}$ we have

$$
\frac{\cos r}{\sin r}=i \frac{z+\frac{1}{z}}{z-\frac{1}{z}}=-i \frac{1+z^{2}}{1-z^{2}}=p(z) ; \quad \text { also, } \quad \frac{\cos r}{\sin r}=\bar{p}(z) .
$$

Calculation of Fourier coefficients gives

$$
(r-\pi)^{2}=\frac{\pi^{2}}{3}+\sum_{k=1}^{\infty} \frac{2}{k^{2}}\left(z^{k}+\bar{z}^{k}\right)=h(z)+\bar{h}(z) .
$$

Now

$p(z) h(z)=-i\left(1+2 \sum_{j=2}^{\infty} I(j) z^{j}\right)\left(\frac{\pi^{2}}{6}+\sum_{k=1}^{\infty} \frac{2}{k^{2}} z^{k}\right)=\frac{-i \pi^{2}}{6}+\sum_{k=1}^{\infty} \beta_{k} z^{k}$

where

Hence

$$
\beta_{k}= \begin{cases}-2 i & k=1 \\ -i\left(4 E(k-2)+\frac{2}{k^{2}}\right) & k>1 .\end{cases}
$$

$$
\frac{(r-\pi)^{2} \cos r}{\sin r}=p(z) h(z)+\bar{p}(z) \bar{h}(z)=\frac{-i \pi^{2}}{3}+\sum_{k=1}^{\infty} \beta_{k}\left(z^{k}-\bar{z}^{k}\right) .
$$


From (5.2.5), we see that

$$
f(r) \sim \gamma_{0}+\sum_{k=1}^{\infty} \gamma_{k}\left(z^{k}+\bar{z}^{k}\right)
$$

where

$$
\begin{aligned}
\gamma_{k}= \begin{cases}-\frac{3}{2}+\frac{1}{8} & k=0 \\
\frac{3}{8}\left(k^{2}+2\right) i k \beta_{k} & k=1(\text { or } k>2) \\
\frac{18 i \beta_{2}}{4}-\frac{1}{16} & k=2\end{cases} \\
= \begin{cases}-\frac{11}{8} & k=0 \\
\frac{9}{4} & k=1, \\
\frac{3 \pi^{2}}{2}+\frac{9}{4}-\frac{1}{16} & k=2 \\
\frac{3}{2}\left(k^{3}+2 k\right) E(k-2)+\frac{3 k}{4}+\frac{3}{2 k} & k>2 .\end{cases}
\end{aligned}
$$

Now

$$
E(k-2)=\frac{\pi^{2}}{8}-\frac{1}{2 k}-\frac{1}{2 k^{2}}-\frac{1}{3 k^{3}}+O\left(k^{-4}\right)
$$

as $k \rightarrow \infty$, so that

$$
\gamma_{k}=\frac{3 \pi^{2}}{16} k^{3}-\frac{3}{4} k^{2}+\frac{3 \pi^{2}}{8} k-2+O\left(\frac{1}{k}\right)
$$

as $k \rightarrow \infty$. The Fourier coefficients $\alpha_{k}=\hat{f}_{k}$ are therefore given by $\alpha_{k}=$ $\gamma_{k}+(3 / 4) k^{2}+2$ and so

$$
\alpha_{k}=\frac{3 \pi^{2}}{16} k^{3}+\frac{3 \pi^{2}}{8} k+O\left(\frac{1}{k}\right)
$$

as $k \rightarrow \infty$. This gives

$$
\alpha_{k}= \begin{cases}\frac{5}{8} & k=0 \\ 5 & k=1 \\ \frac{3 \pi^{2}}{2}+7+\frac{3}{16} & k=2 \\ \frac{3}{2}\left(k^{3}+2 k\right) E(k-2)+\frac{3}{4}\left(k^{2}+k+\frac{2}{k}\right)+2 & k>2\end{cases}
$$

so that $\alpha_{k}>0$ for all $k>1$.

5.3. The Hessian of $\log \operatorname{det} L$ at $S^{n}$. Let $g_{0}$ be the standard metric on $S^{n}$ where $n$ is odd. As usual $r$ denotes the distance between points $x, y \in S^{n}$. Green's function for $L_{0}$ is

$$
G(r)=K\left(L_{0}^{-1}, x, y\right)=\frac{C_{n}}{\sin ^{n-2}(r / 2)}, \quad C_{n}=\frac{1}{(n-2) V_{n-1}} .
$$


Since this has singular parity as $r \downarrow 0,\left(L_{0}^{-1}\right)_{\text {reg }}=0$. Furthermore, $R_{i j}=2 \delta_{i j}$. From this we see that (1.3.11) reduces to

$$
\text { Hess } \log \operatorname{det} L(A, A)=-\mathrm{TR} A_{i j} D_{i j}^{2} L_{0}^{-1} A_{k \ell} D_{k \ell}^{2} L_{0}^{-1}
$$

when $A \in S_{0}^{2} T^{*} S^{n}$ with $A_{i j, j}=0$.

Lemma 5.3.1. Suppose that $A \in C^{\infty}\left(S_{0}^{2} T^{*} S^{2}\right)$, where the trace is with respect to the standard metric. Then when $a(x, v)=A_{i j}(x) v_{i} v_{j}$,

$$
\begin{aligned}
\operatorname{TR} A_{i j} & D_{i j}^{2} L_{0}^{-1} A_{k \ell} D_{k \ell}^{2} L_{0}^{-1} \\
& =(-1)^{(n+1) / 2} \frac{\pi}{V_{n}^{2}} \int_{S S^{3}} \sum_{k=0}^{\infty} \alpha_{k}\left|\hat{a}_{k}(x, v)\right|^{2} d \sigma_{n-1}(v) d \sigma_{n}(x),
\end{aligned}
$$

where $\alpha_{k}>0$ for all $k$, so that (5.3.1) is nonzero unless $A_{k \ell}=0$, and has the same sign as $(-1)^{(n-1) / 2}$.

Proof. By Lemma 5.1.2, (5.3.2) holds where $\alpha_{k}$ are the Fourier coefficients of $(-1)^{(n+1) / 2} f(r)=(-1)^{(n+1) / 2}(T G(r))^{2} \sin ^{2} r$. We will follow the strategy outlined below Lemma 5.1.4 to compute these Fourier coefficients.

$$
T G(r)=\sin r \frac{d}{d r} \frac{1}{\sin r} \frac{d}{d r} G(r)=C_{n}^{\prime} \frac{\cos ^{2}(r / 2)}{\sin ^{n}(r / 2)},
$$

and

$$
f(r)=\sin ^{n-1} r(T G(r))^{2}=C_{n}^{\prime \prime} \cos ^{2}(r / 2)\left(\frac{\cos (r / 2)}{\sin (r / 2)}\right)^{n+1}, \quad C_{n}^{\prime \prime}>0 .
$$

Now if $z=e^{i r}$,

$$
(-1)^{(n+1) / 2}\left(\frac{\cos (r / 2)}{\sin (r / 2)}\right)^{n+1}=\left(\frac{1+z}{1-z}\right)^{n+1} .
$$

Lemma 5.3.2. For fixed $n$, let $p(k)$ be the coefficients defined by

$$
\left(\frac{1+z}{1-z}\right)^{n+1}=\sum_{k=0}^{\infty} p(k) z^{k}
$$

Then $p(k)>0$ for all $k$. Moreover for $k>n+1$,

$$
p(k)=a_{n} k^{n}+a_{n-2} k^{n-2}+\cdots+a_{1} k, \quad \text { where } a_{j}>0 \text { for all } j .
$$

Proof.

$$
\begin{aligned}
\left(\frac{1}{1-z}\right)^{n+1} & =\sum_{k=0}^{\infty} \frac{(k+1)(k+2) \ldots(k+n)}{n !} z^{k} \\
(1+z)^{n+1} & =\sum_{\ell=0}^{n+1}\left(\begin{array}{c}
n+1 \\
\ell
\end{array}\right) z^{\ell} .
\end{aligned}
$$


Since the coefficients of both of these power series are positive, so are the coefficients $p(k)$ in the product. Moreover when $k>n+1, p(k)$ is equal to

$$
\begin{aligned}
& \frac{1}{n !} \sum_{\ell=0}^{n+1}\left(\begin{array}{c}
n+1 \\
\ell
\end{array}\right) \prod_{j=1}^{n}(k+j-\ell) \\
&=\frac{1}{n !} \sum_{\ell=0}^{(n+1) / 2}\left(\begin{array}{c}
n+1 \\
\ell
\end{array}\right)\left(\prod_{j=1-\ell}^{n-\ell}(k+j)+\prod_{j=1-\ell}^{n-\ell}(k-j)\right)
\end{aligned}
$$

but since $n$ is odd,

$$
\prod_{j=1-\ell}^{n-\ell}(k+j)+\prod_{j=1-\ell}^{n-\ell}(k-j)
$$

is a polynomial having the form given in (5.3.4).

Now we see that when $z=e^{i r}$,

$$
\cos ^{2}(r / 2)=\left(\frac{z^{1 / 2}+z^{-1 / 2}}{2}\right)^{2}=\frac{1}{4}\left(z+2+z^{-1}\right),
$$

so that

and

$$
\cos ^{2}(r / 2)=\frac{1}{4}(z+1)+\frac{1}{4}(1+\bar{z})
$$

$$
\begin{aligned}
& (-1)^{(n+1) / 2} \cos ^{2}(r / 2)\left(\frac{\cos (r / 2)}{\sin (r / 2)}\right)^{n+1} \\
& \quad \frac{1}{4}(z+1) \sum_{k=0}^{\infty} p(k) z^{k}+\frac{1}{4}(1+\bar{z}) \sum_{k=0}^{\infty} p(k) \bar{z}^{k} \\
& =\frac{1}{4}\left(p(0)+\sum_{k=1}^{\infty}(p(k)+p(k-1)) z^{k}\right) \\
& +\frac{1}{4}\left(p(0)+\sum_{k=1}^{\infty}(p(k)+p(k-1)) \bar{z}^{k}\right) \\
& =c(0)+\sum_{k=1}^{\infty} c(k)\left(z^{k}+\bar{z}^{k}\right) .
\end{aligned}
$$

Now let us examine the coefficient $c(k)$ for large $k$.

$$
\begin{aligned}
c(k)= & \frac{1}{4}(p(k)+p(k-1)) \\
= & \frac{1}{4}\left(a_{n} k^{n}+a_{n-2} k^{n-2}+\cdots+a_{1} k\right. \\
& \left.\quad+a_{n}(k-1)^{n}+a_{n-2}(k-1)^{n-2}+\cdots+a_{1}(k-1)\right) \\
= & b_{n} k^{n}+b_{n-1} k^{n-1}+\cdots+b_{0}
\end{aligned}
$$


where

$$
\begin{cases}b_{n}>0 & n \text { odd } \\ b_{n}<0 & n \text { even. }\end{cases}
$$

Now for every $k \geq 0$, set

$$
e(k)=-b_{n-1} k^{n-1}-b_{n-3} k^{n-3}-\cdots-b_{0}
$$

so that $e(k)$ is positive. Then

$$
\begin{aligned}
(-1)^{(n+1) / 2} \cos ^{2}(\theta / 2)\left(\frac{\cos (r / 2)}{\sin (r / 2)}\right)^{n+1} \sim & c(0)+e(0) \\
& +\sum_{k=1}^{\infty}(c(k)+e(k))\left(z^{k}+\bar{z}^{k}\right)
\end{aligned}
$$

and $(-1)^{(n+1) / 2} \hat{f}_{k}=C_{n}^{\prime \prime}(c(k)+e(k))>0$.

\section{Saddle points for $\operatorname{det}^{\prime} \Delta$}

In this section we show that the $(4 m+3)$-sphere with $m=1,2, \ldots$, is a saddle point for $F$ under conformal deformations, by proving Lemma 1.3.12 which states that if $n>3$ is odd and $\phi \in \mathcal{H}_{2}$, then

$$
\begin{aligned}
\frac{(n+2)(n-2)}{2} & \int_{S^{n}} \bar{\phi}^{2} d \mu_{n}-\frac{(n-2)^{2}}{4} \operatorname{TR} \phi_{i i} \bar{\Delta}_{0}^{-1} \phi_{k k} \bar{\Delta}_{0}^{-1} \\
= & \frac{2(n-2)\left(-2+(n-2)(n+1) \sum_{j=2}^{n-1} \frac{1}{j}\right)}{(n-3) n} \int_{S^{n}} \phi^{2} d \mu_{n} .
\end{aligned}
$$

The left side of (6.1.1) is Hess $F\left(2 \phi g_{0}, 2 \phi g_{0}\right)$. The space $\mathcal{H}_{k}$ has dimension

$$
d_{k}=\frac{(k+1)(k+2) \ldots(k+n-2)(2 k+n-1)}{(n-1) !}
$$

and the eigenvalue of $\Delta$ on $\mathcal{H}_{k}$ is

$$
\lambda_{k}=k(k+n-1) .
$$

Write $Z_{n}(s)$ for the zeta function

$$
Z(s)=\sum_{j=1}^{\infty} d_{j} \lambda_{j}^{-s}
$$

defined via analytic continuation for $\Re s \leq n / 2$.

Define $\tilde{\lambda}_{k}^{-1}$ to be the eigenvalues of $\bar{\Delta}_{0}^{-1}$, so that

$$
\frac{1}{\tilde{\lambda}_{k}}= \begin{cases}-Z(1), & k=0 \\ 1 / \lambda_{k}, & k \geq 1\end{cases}
$$


(We do not rule out the possibility that $Z(1)=0$.) Define the measure $d \nu_{n}$ on $[-1,1]$ by

$$
d \nu_{n}(t)=\frac{1}{v}\left(1-t^{2}\right)^{(n / 2)-1} d t, \quad v=\frac{(n-1) ! \pi}{\left.2^{n-1}((n-1) / 2) !\right)^{2}} .
$$

The normalization ensures that $\nu_{n}([-1,1])=1$. Fix $n$, and for $k=0,1,2, \ldots$, let $P_{k}(t)$ be the orthogonal polynomials on $[-1,1]$ with respect to this measure normalized so that

$$
\int_{-1}^{1} P_{k}^{2}(t) d \nu_{n}(t)=d_{k}
$$

These are eigenfunctions for

$$
\Delta_{n}=-\left(1-t^{2}\right)^{1-(n / 2)} \frac{d}{d t}\left(1-t^{2}\right)^{n / 2} \frac{d}{d t} .
$$

The orthogonal projection $\Pi_{k}$ of $L^{2}\left(S^{n}\right)$ onto $\mathcal{H}_{k}$ has kernel $K(x, y)=P_{k}(x \cdot y)$. To prove Lemma 1.3.12, we just have to check that (6.1.1) holds for some $\phi \in \mathcal{H}_{2}$. Fix a point $x_{0} \in S^{n}$ and set

$$
\begin{array}{rlrl}
\phi(x) & =\frac{1}{\lambda_{2}} P_{2}\left(x, x_{0}\right) & & \text { so that } \\
\phi_{k k}(x) & =P_{n, 2}\left(x, x_{0}\right), \quad & \int \bar{\phi}^{2} d \mu_{n}=\frac{d_{2}}{\lambda_{2}^{2}}=\frac{n(n+3)}{8(n+1)^{2}} .
\end{array}
$$

From this we can calculate the first term in (6.1.1), and Lemma 1.3.12 follows if we show that

$$
\operatorname{TR} \phi_{k k} \bar{\Delta}^{-1} \phi_{j j} \bar{\Delta}^{-1}=\frac{(n+3)\left(n^{2}+n-4-4(n+1) \sum_{j=2}^{n-1} \frac{1}{j}\right)}{4(n-3)(n+1)^{2}} .
$$

\section{Now}

$$
\begin{aligned}
\operatorname{trace} \phi_{i i} \Pi_{k} \phi_{j j} \Pi_{\ell} & =\int_{S^{n} \times S^{n}} \phi_{i i}(x) P_{k}(x \cdot y) \phi_{j j}(y) P_{\ell}(x \cdot y) d \mu_{n} d \mu_{n} \\
& =\int_{S^{n} \times S^{n}} P_{2}\left(x \cdot x_{0}\right) P_{k}(x \cdot y) P_{2}\left(y, x_{0}\right) P_{\ell}(x \cdot y) d \mu_{n} d \mu_{n} .
\end{aligned}
$$

By symmetry this does not depend on $x_{0}$ and we can average over $x_{0} \in S^{n}$ to obtain

$$
\int_{S^{n} \times S^{n}} P_{2}(x \cdot y) P_{k}(x \cdot y) P_{\ell}(x \cdot y) d \mu_{n} d \mu_{n}=\int_{-1}^{1} P_{2} P_{k} P_{\ell} d \nu_{n} .
$$

Set $p=(n-1) / 2$, and $B=\Delta^{2}+p^{2}$ which has eigenvalue $(j+p)^{2}$ on $\mathcal{H}_{j}$, and set

$$
C(j, m, \ell)=\int_{-1}^{1} P_{j} P_{j+m} P_{\ell} d \nu_{n}, \quad F(j, m, \ell)=\frac{C(j, m, \ell)}{\lambda_{j} \lambda_{j+m}}
$$


Then

$$
\begin{aligned}
& \operatorname{TR} \phi_{k k} \bar{\Delta}^{-1} \phi_{j j} \bar{\Delta}^{-1}=\left.\operatorname{trace} \phi_{k k} \bar{\Delta}^{-1} B^{z / 2} \phi_{j j} \bar{\Delta}^{-1}\right|_{z=0} ^{\text {mer }} \\
& =\left.\sum_{j, k=0}^{\infty} \frac{\operatorname{trace} \phi_{i i} \Pi_{j} \phi_{\ell \ell} \Pi_{k}}{\tilde{\lambda}_{j} \tilde{\lambda}_{k}}(j+p)^{z}\right|_{z=0} ^{\text {mer }}=\left.\sum_{j, k=0}^{\infty} \frac{\int P_{j} P_{k} P_{2} d \nu_{n}}{\tilde{\lambda}_{j} \tilde{\lambda}_{k}}(j+p)^{z}\right|_{z=0} ^{\text {mer }} \\
& \left.=\frac{2}{\tilde{\lambda}_{0}} \frac{d_{2}}{\lambda_{2}}+\left.\sum_{j, k=1}^{\infty} \frac{\int P_{j} P_{k} P_{2} d \nu_{n}}{\lambda_{j} \lambda_{k}}(j+p)^{z}\right|_{z=0} ^{\text {mer }}+\sum_{j=3}^{\infty} F(j,-2,2)(j+p)^{z}\right)\left.\right|_{z=0} ^{\text {mer }} \\
& =\frac{2}{\tilde{\lambda}_{0}} \frac{d_{2}}{\lambda_{2}}+\left(\sum_{j=1}^{\infty} F(j, 0,2)(j+p)^{z}+\sum_{j=1}^{\infty} F(j, 2,2)(j+p)^{z}\right. \\
& =\frac{2}{\tilde{\lambda}_{0}} \frac{d_{2}}{\lambda_{2}}+\sum_{j=1}^{2} F(j, 0,2)+\sum_{j=1}^{2} F(j, 2,2) \\
& +\left.\sum_{j=3}^{\infty}(F(j, 0,2)+F(j, 2,2)+F(j,-2,2))(j+p)^{z}\right|_{z=0} ^{\text {mer }} .
\end{aligned}
$$

The final sum in (6.1.5) and the quantity $1 / \tilde{\lambda}_{0}=Z(1)$ both involve a zeta regularization. In order to evaluate these two quantities we make use of the following lemma.

Lemma 6.1.1. Suppose that $G$ is an even polynomial; that is, $G(-j)=$ $G(j)$, and $\ell>1$. Then

$$
\left.\sum_{\substack{j \neq \ell \\ 1 \leq j<\infty}} \frac{G(j)}{j^{2}-\ell^{2}} j^{z}\right|_{z=0} ^{\text {mer }}=\frac{G(0)}{2 \ell^{2}}+\frac{G(\ell)}{4 \ell^{2}}-\frac{G^{\prime}(\ell)}{2 \ell} .
$$

Proof. Set

$$
H(j)= \begin{cases}\frac{G(j)-G(\ell)}{j^{2}-\ell^{2}} & j^{2} \neq \ell \\ \frac{G^{\prime}(j)}{2 \ell} & j^{2}=\ell^{2} .\end{cases}
$$

Then $H$ is an even polynomial and since $\zeta(-2 k)=0$ for $k=1,2, \ldots$, and $\zeta(0)=-1 / 2$, we see that

$$
\begin{aligned}
\left.\sum_{j=1}^{\infty} H(j) j^{z}\right|_{z=0} ^{\text {mer }} & =\left.\sum_{j=1}^{\infty} \sum_{k=0}^{\operatorname{deg} H} \frac{H^{(2 k)}(0)}{(2 k) !} j^{2 k+z}\right|_{z=0} ^{\text {mer }} \\
& =\sum_{k=0}^{\operatorname{deg} H} \frac{H^{(2 k)}(0)}{(2 k) !} \zeta(-2 k)=-\frac{H(0)}{2}=\frac{G(0)}{2 \ell^{2}}-\frac{G(\ell)}{2 \ell^{2}}
\end{aligned}
$$


Hence

(6.1.6)

$$
\begin{aligned}
\sum_{j=1}^{\ell-1} \frac{G(j)}{j^{2}-\ell^{2}} & +\frac{G^{\prime}(\ell)}{2 \ell}+\left.\sum_{j=\ell+1}^{\infty} \frac{G(j)}{j^{2}-\ell^{2}} j^{z}\right|_{z=0} ^{\text {mer }} \\
& =\left.\sum_{j=1}^{\infty} H(j) j^{z}\right|_{z=0} ^{\text {mer }}+G(\ell)\left(\sum_{j=1}^{\ell-1} \frac{1}{j^{2}-\ell^{2}}+\sum_{j=\ell+1}^{\infty} \frac{1}{j^{2}-\ell^{2}}\right) \\
& =\frac{G(0)}{2 \ell^{2}}-\frac{G(\ell)}{2 \ell^{2}}+\frac{3 G(\ell)}{4 \ell^{2}}
\end{aligned}
$$

From Lemma 6.1.1, we get the following.

LEMMA 6.1.2.

$$
Z(1)=-\frac{1}{n-1} \sum_{j=1}^{n-1} \frac{1}{j}
$$

Proof. As before, set $p=(n-1) / 2$, and $B=\Delta^{2}+p^{2}$ which has eigenvalue $(j+p)^{2}$ on $\mathcal{H}_{j}$. Using the canonical trace, we have

$$
\begin{aligned}
Z(1) & =\left.\operatorname{TR} \Delta^{-1} B^{z / 2}\right|_{z=0} ^{\mathrm{mer}}=\left.\sum_{j=1}^{\infty} \frac{d_{j}}{\lambda_{j}}(j+p)^{z}\right|_{z=0} ^{\mathrm{mer}} \\
& =\left.\frac{2}{(n-1) !} \sum_{j=1}^{\infty} \frac{\prod_{k=0}^{p-1}\left((j+p)^{2}-k^{2}\right)}{(j+p)^{2}-p^{2}}(j+p)^{z}\right|_{z=0} ^{\mathrm{mer}} \\
& =\left.\frac{2}{(n-1) !} \sum_{j=p+1}^{\infty} \frac{\prod_{k=0}^{p-1}\left(j^{2}-k^{2}\right)}{j^{2}-p^{2}} j^{z}\right|_{z=0} ^{\mathrm{mer}} .
\end{aligned}
$$

Now, applying Lemma 6.1.1 with $\ell=p$ and

$$
G(j)=\frac{2}{(n-1) !} \prod_{k=0}^{p-1}\left(j^{2}-k^{2}\right)=\frac{2 j(j-p+1) \ldots(j+p-1)}{(n-1) !},
$$

we find that

$$
\begin{array}{ll}
G(j) & =0 \quad \text { for }|j|<p, \\
G(p) & =1, \\
G^{\prime}(p) & =\sum_{j=1}^{n-1} \frac{1}{j}+\frac{1}{n-1},
\end{array}
$$


and so

$$
\left.\sum_{j=p+1}^{\infty} \frac{G(j)}{j^{2}-\ell^{2}} j^{z}\right|_{z=0} ^{\text {mer }}=\frac{G(p)}{4 p^{2}}-\frac{G^{\prime}(p)}{2 p}=-\frac{1}{n-1} \sum_{j=1}^{n-1} \frac{1}{j}
$$

From Lemma 6.1.2, we can compute the first term in (6.1.5) and get

$$
\frac{2}{\tilde{\lambda}_{0}} \frac{d_{2}}{\lambda_{2}}=\frac{n(n+3)}{2(n-1)(n+1)} \sum_{j=1}^{n-1} \frac{1}{j}
$$

To compute the other terms in (6.1.5), standard techniques give the recurrence relation

$$
x P_{k}(x)=\frac{k+1}{2 k+n+1} P_{k+1}+\frac{k+n-2}{2 k+n-3} P_{k-1} .
$$

From this it can be deduced that

$$
P_{2}(t)=\frac{1}{2}(n+3)\left((n+1) x^{2}-1\right)
$$

and the linearization coefficients defined in (6.1.4), are computed as in $[\mathrm{V}$, 9.11.6]:

$$
\begin{aligned}
C(j, 0,2) & =\frac{n+3}{2(n-2) !}(j) \cdots(j+p-2)(j+p)^{2}(j+2) \cdots(j+n-1) & & j \geq 0, \\
C(j, 2,2) & =\frac{(n+1)(n+3)}{4(n-1) !}(j+1) \cdots(j+p)(j+p+2) \cdots(j+n), & & j \geq 0, \\
C(j,-2,2) & =\frac{(n+1)(n+3)}{4(n-1) !}(j-1) \cdots(j+p-2)(j+p) \cdots(j+n-2), & & j \geq 2 .
\end{aligned}
$$

Hence

$$
\begin{aligned}
\sum_{j=1}^{2} F(j, 0,2)+\sum_{j=1}^{2} F(j, 2,2)= & \frac{n+1}{n}+\frac{(n-1) n(n+3)^{2}}{4(n+1)^{2}(n+5)} \\
& +\frac{(n+1)^{2}}{6(n+2)}+\frac{n(n+1)(n+2)}{32(n+5)}
\end{aligned}
$$

We use Lemma 6.1.1 to compute the final sum in (6.1.5). First we write this term in the form

$$
\left.\sum_{j=p+3}^{\infty}(F(j-p, 0,2)+F(j-p, 2,2)+F(j-p,-2,2)) j^{z}\right|_{z=0} ^{\text {mer }} .
$$


Using the formulas for the linearization coefficients we get

$$
\begin{aligned}
& F(j-p, 0,2)+F(j-p, 2,2)+F(j-p,-2,2) \\
& =\frac{n+3}{2(n-1) !}\left(\frac{2 p(j-p) \ldots(j-2) j^{2}(j+2) \ldots(j+p)}{(j-p)^{2}(j+p)^{2}}\right. \\
& +\frac{(p+1)(j-p+1) \ldots j(j+2) \ldots(j+p+1)}{(j-p)(j+p)(j-p+2)(j+p+2)} \\
& \left.\quad+\frac{(p+1)(j-p-1) \ldots(j-2) j \ldots(j+p-1)}{(j-p)(j+p)(j-p-2)(j+p-2)}\right) \\
& =\frac{n+3}{(n-1) !} \frac{(j-p+1) \ldots(j+p-1)}{(j-p)(j+p)(j-p-2)(j+p-2)(j-p+2)(j+p+2)} j Q(j)
\end{aligned}
$$

where

$$
Q(j)=n j^{2}-\frac{n(n+3)^{2}}{4}+8
$$

Writing

$$
G(j)=\frac{n+3}{(n-1) !}(j-p+1)(j-p+3) \ldots(j+p-3)(j+p-1) j Q(j),
$$

we see that (6.1.10) equals

$$
\begin{aligned}
& \sum_{j=p+3}^{\infty} \frac{G(j)}{\left(j^{2}-p^{2}\right)\left(j^{2}-(p+2)^{2}\right)} \\
& \quad=\frac{1}{(p+2)^{2}-p^{2}}\left(\sum_{j=p+3}^{\infty} \frac{G(j)}{\left(j^{2}-(p+2)^{2}\right)}-\sum_{j=p+3}^{\infty} \frac{G(j)}{\left(j^{2}-p^{2}\right)}\right)
\end{aligned}
$$

to which we can apply Lemma 6.1.1. Using the fact that $G(j)=0$ for $0 \leq j \leq$ $p-3$ and $j=p-1$, we find that (6.1.10) equals

$$
\begin{gathered}
\frac{1}{4(p+1)}\left(-\frac{G(p-2)}{(p-2)^{2}-(p+2)^{2}}-\frac{G(p)}{\left(p^{2}-(p+2)^{2}\right)}-\frac{G(p+1)}{(p+1)^{2}-(p+2)^{2}}\right. \\
+\frac{G(p+2)}{4(p+2)^{2}}-\frac{G^{\prime}(p+2)}{2(p+2)}+\frac{G(p-2)}{(p-2)^{2}-p^{2}}+\frac{G(p+1)}{(p+1)^{2}-p^{2}} \\
\left.+\frac{G(p+2)}{(p+2)^{2}-p^{2}}-\frac{G(p)}{4 p^{2}}+\frac{G^{\prime}(p)}{2 p}\right)
\end{gathered}
$$


We evaluate this using the values

$$
\begin{aligned}
G(p-2)= & \frac{2(n+1)(n+3)}{(n-1)(n-3)}, \quad G(p)=-\frac{(n+3)\left(n^{2}+n-4\right)}{2(n-3)}, \\
G(p+1)= & -\frac{(n+1)(n+3)(n+4)}{6}, \quad G(p+2)=\frac{n(n+3)^{2}}{2(n-1)}, \\
G^{\prime}(p)= & \frac{(n-1) n(n+3)}{4(n-3)} \\
& -\frac{(n+3)\left(n^{2}+n-4\right)}{2(n-3)}\left(\sum_{j=2}^{n-1} \frac{1}{j}+\frac{1}{2}-\frac{1}{n-3}+\frac{1}{n-1}\right), \\
G^{\prime}(p+2)= & \frac{n(n+3)^{2}}{2(n-1)}\left(\sum_{j=3}^{n-2} \frac{1}{j}-\frac{1}{4}+\frac{1}{n}+\frac{2}{n+3}+\frac{n(n+3)}{8} .\right),
\end{aligned}
$$

and we find that (6.1.7), (6.1.9) and (6.1.10) sum to the right-hand side of (6.1.3).

University of California San Diego, La Jolla, CA

E-mail address: okikiolu@math.ucsd.edu

\section{REFERENCES}

[Br] T. Branson, Sharp inequalities, the functional determinant, and the complementary series, Trans. A.M.S. 347 (1995), 3671-3742.

[BFK1] D. Burghelea, L. Friedlander, and T. Kappeler, On the determinant of elliptic differential and finite difference operators in vector bundles over $S^{1}$, Comm. Math Phys. 138 (1991), 1-18.

[BFK2] Meyer-Vietoris type formula for determinants of elliptic differential operators, J. Funct. Anal. 107 (1992), 34-65.

[BO1] T. Branson and B. Ørstead, Conformal geometry and global invariants, Differential Geom. and Appl. 1 (1991), 279-308.

[BO2] Explicit functional determinants in four dimensions, Proc. A.M.S. 113 (1991), 669-682.

[BCY] T. Branson, S.-Y. A. Chang, and P. YAng, Estimates and extremals for zeta function determinants on four-manifolds, Commun. Math. Phys. 149 (1992), 241-262.

[CQ1] S.-Y. A. ChANG and J. QING, Zeta functional determinants on manifolds with boundary, Math. Res. Lett. 3 (1996), 1-17.

[CQ2] The zeta functional determinants on manifolds with boundary. I. The formula. J. Funct. Anal. 147 (1997), 327-362.

[CQ3] The zeta functional determinants on manifolds with boundary. II. Extremal metrics and compactness of isospectral set, J. Funct. Anal. 147 (1997), 363-399.

[CY] S.-Y. A. ChAng and P. YAng, Extremal metrics of zeta function determinants on 4-manifolds, Ann. of Math. 142 (1995), 171-212.

[Chi] P. Chiu, Height of flat tori, Proc. A.M.S. 125 (1997), 723-730.

[Cho] B. Chow, The Ricci flow on the 2-sphere, J. Differential Geom. 33 (1991), 325-334.

[Eb] D. EBin, On the space of Riemannian metrics, Bull. A.M.S. 75 (1968), 1001-1003. 
[Fo1] R. Forman, Functional determinants and geometry, Invent. Math. 88 (1987), 447493; Erratum ibid. 108 (1992), 453-454.

[Fo2] Determinant of the Laplacian on graphs, Topology 32 (1993), 35-46.

[Fo3] Determinants, finite-difference operators and boundary value problems, Commun. Math. Phys. 147 (1992), 485-526.

[Ha] R. Hamilton, The Ricci flow on surfaces, in Mathematics and General Relativity (Santa Cruz, 1986), 237-262, Contemp. Math. 71, A.M.S., Providence, RI (1988).

[Ka] C. Kassel, Le résidu non commutatif (d'apres M. Wodzicki), Sém. Bourbaki, Exp. No. 708 (1988-89), 199-229.

[Gui] V. Gulllemin, A new proof of Weyl's formula on the asymptotic distribution of eigenvalues, Adv. in Math. 55 (1985), 131-160.

[Gur] M. Gursky, Uniqueness of the functional determinant, Commun. Math. Phys. 189 (1997), 655-665.

[KV] M. Kontseivich and S. Vishik, Geometry of determinants of elliptic operators, in Funct. Anal. on the Eve of the $21^{\text {st }}$ Century, Vol. 1, Birkhäuser Boston, MA, Progr. Math. 131 (1995), 173-197.

[LP] J. Lee and T. PArker, The Yamabe problem, Bull. A.M.S. 17 (1987), 37-91.

[Ok1] K. Oкıкіоц, The Campbell-Hausdorff theorem for elliptic operators and a related trace formula, Duke Math. J. 79 (1995), 687-722.

[Ok2] The multiplicative anomaly for determinants of elliptic operators, Duke Math. J. 79 (1995), 723-750.

[Ok3] Critical metrics for spectral zeta functions, preprint.

[Ok4] A note on the space of metrics modulo diffeomorphisms, in preparation.

[On] E. ONOFRI, On the positivity of the effective action in a theory of random surfaces, Commun. Math. Phys. 86 (1982), 321-326.

[OPS1] B. Osgood, R. Phillips, and P. SARnak, Extremals of determinants of Laplacians, J. Funct. Anal. 80 (1988), 148-211.

[OPS2] Compact isospectral sets of surfaces, J. Funct. Anal. 80 (1988), 212-234.

[OPS3] Moduli space, heights and isospectral sets of plane domains, Ann. of Math. 129 (1989), 293-362.

[PR] T. PArker and S. Rosenberg, Invariants of conformal Laplacians, J. Differential Geom. 25 (1987), 199-222.

[Po] A. Polyakov, Quantum geometry of bosonic strings, Phys. Lett. B 103 (1981), 207-210.

[RS] D. RAY and I. Singer, $R$-torsion and the Laplacian on Riemannian manifolds, Adv. in Math. 7 (1971), 145-210.

[Ri] K. Richardson, Critical points of the determinant of the Laplace operator, J. Funct. Anal. 122 (1994), 52-83.

[Sp] M. SpIvak, A Comprehensive Introduction to Differential Geometry, Vol. 1, Publish or Perish, Wilmington, Del. (1979).

[Ta] M. TaYlor, Partial Differential Equations I., Basic Theory, Appl. Math. Sci. 115, Springer-Verlag, New York, 1996.

[Tr] F. Trèves, Basic Linear Partial Differential Equations, Pure and Appl. Math. 62, Academic Press, New York (1975).

[Vi] N. Vilenkin, Special Functions and the Theory of Group Representations, Transl. Math. Mono. 22, A.M.S., Providence, RI (1968).

[Wo] M. Wodzicki, Noncommutative residue. I. Fundamentals, in K-Theory, Arithmetic and Geometry (Moscow, 1984-1986), Lecture Notes in Math. 1289 (1987), 320-399, Springer-Verlag, New York.

(Received September 20, 1999) 